\title{
Bifurcations of a periodically forced microbial continuous culture model with restrained growth rate
}

Jingli Ren, and Qigang Yuan

Citation: Chaos 27, 083124 (2017);

View online: https://doi.org/10.1063/1.5000152

View Table of Contents: http://aip.scitation.org/toc/cha/27/8

Published by the American Institute of Physics

\section{Articles you may be interested in}

Constructing an autonomous system with infinitely many chaotic attractors

Chaos: An Interdisciplinary Journal of Nonlinear Science 27, 071101 (2017); 10.1063/1.4986356

A new chaotic oscillator with free control

Chaos: An Interdisciplinary Journal of Nonlinear Science 27, 083101 (2017); 10.1063/1.4997051

Resilience of epidemics for SIS model on networks

Chaos: An Interdisciplinary Journal of Nonlinear Science 27, 083105 (2017); 10.1063/1.4997177

Delay-induced locking in bursting neuronal networks

Chaos: An Interdisciplinary Journal of Nonlinear Science 27, 083114 (2017); 10.1063/1.4998927

Stationary distribution of a stochastic SIS epidemic model with double diseases and the Beddington-DeAngelis incidence

Chaos: An Interdisciplinary Journal of Nonlinear Science 27, 083126 (2017); 10.1063/1.4986838

Detection of coupling delay: A problem not yet solved

Chaos: An Interdisciplinary Journal of Nonlinear Science 27, 083109 (2017); 10.1063/1.4997757 


\title{
Bifurcations of a periodically forced microbial continuous culture model with restrained growth rate
}

\author{
Jingli Ren ${ }^{1,2, a)}$ and Qigang Yuan ${ }^{1}$ \\ ${ }^{1}$ School of Mathematics and Statistics, Zhengzhou University, Zhengzhou 450001, China \\ ${ }^{2}$ State Key Laboratory of Nonlinear Mechanics, Institute of Mechanics, Chinese Academy of Sciences, \\ Beijing 100190, China
}

(Received 3 April 2017; accepted 14 August 2017; published online 29 August 2017)

\begin{abstract}
A three dimensional microbial continuous culture model with a restrained microbial growth rate is studied in this paper. Two types of dilution rates are considered to investigate the dynamic behaviors of the model. For the unforced system, fold bifurcation and Hopf bifurcation are detected, and numerical simulations reveal that the system undergoes degenerate Hopf bifurcation. When the system is periodically forced, bifurcation diagrams for periodic solutions of period-one and period-two are given by researching the Poincaré map, corresponding to different bifurcation cases in the unforced system. Stable and unstable quasiperiodic solutions are obtained by NeimarkSacker bifurcation with different parameter values. Periodic solutions of various periods can occur or disappear and even change their stability, when the Poincaré map of the forced system undergoes Neimark-Sacker bifurcation, flip bifurcation, and fold bifurcation. Chaotic attractors generated by a cascade of period doublings and some phase portraits are given at last. Published by AIP Publishing. [http://dx.doi.org/10.1063/1.5000152]
\end{abstract}

The microbiological fermentation technique is widely applied in many fields for its economic importance. It is also investigated due to the complex behaviours observed during the process. We study bifurcations of the microbial continuous culture model with two types of dilution rates: the steady dilution rate and periodically forced dilution rate. For the steady dilution rate, we prove that the model undergoes fold bifurcation and Hopf bifurcation. When the dilution rate is periodically forced, we find that the bifurcations of the equilibria of the unforced system can be extended to the forced system as bifurcations of periodic solutions. Furthermore, periodic perturbation can give rise to complex dynamics, such as quasiperiodic solutions, periodic solutions of various periods, and chaos. In addition, the various periodic solutions can well explain the oscillation phenomena observed in laboratory experiments.

\section{INTRODUCTION}

The microbiological fermentation technique, as a mature and practical technology, is used to produce useful materials such as industrial raw materials and pharmaceutical products. Among various microbial production methods, the microbial continuous culture of glycerol to 1, 3-propanediol (1,3-PD) is particularly attractive to industry, because of renewable feedback and potential use of 1, 3-propanediol. It is a complicated biochemical reaction process, in which the substrate and product are transported or diffused through the cell membrane, and a series of sequential and branched reaction are carried out by catalysis of enzymes in cells. ${ }^{1}$

Many experiments and numerical simulations have been done for microbial fermentations, see Refs. 2-5 and the

\footnotetext{
${ }^{a)}$ Author to whom correspondence should be addressed: renjl@zzu.edu.cn
}

references therein. Based on the experimental results in Ref. 5, the authors established a parameter identification model ${ }^{6}$ and then discussed the equilibria and their stability. ${ }^{7}$ However, some complex behaviours, such as oscillation phenomena ${ }^{4,8}$ in a microbial continuous culture process, are not explained in Refs. 6 and 7. To better understand the phenomena, we study the complex dynamics of continuous culture by using the bifurcation theory and continuation technique for the nonautonomous system. For simplicity, by-products in a continuous culture process such as ethyl alcohol and acetic acid are not considered. Therefore, the model in Ref. 6 can be rewritten as

$$
\left\{\begin{array}{l}
\frac{d x}{d t}=x(\mu-D), \\
\frac{d y}{d t}=D\left(a_{0}-y\right)-x\left(n_{s}+\frac{\mu}{Y_{s}}\right) \\
\frac{d z}{d t}=x\left(n_{p}+Y_{p} \mu\right)-D z
\end{array}\right.
$$

where $x, y$, and $z$ are the concentration of the microorganism (biomass), glycerol, and 1,3-PD in the culture container, respectively. The coefficients in model (1) are illustrated in Table I.

In (1), the growth rate $\mu$ is a function of the variables $y$ and $z$, which plays an important role in the process for determining the output of the product. There are several types of growth rates for microorganism and cultural conditions, such as Contios type $^{9}$ for limiting nutrient conditions and Andrews type ${ }^{10}$ for the high concentration of the initial substrate. Considering that the excessively high concentration of the glycerol and 1,3-PD restrains the growth of the microorganism, the growth rate can be expressed as

$$
\mu=\mu_{\max } \frac{y}{y+K}\left(1-\frac{y}{c}\right)\left(1-\frac{z}{d}\right),
$$


TABLE I. The mean of the coefficients in the system.

\begin{tabular}{lc}
\hline \hline$x$ & Biomass concentration $\left(\mathrm{mmol} \mathrm{l}^{-1}\right)$ \\
$y$ & Extracellular glycerol concentration in the reactor $\left(\mathrm{mmol}^{-1}\right)$ \\
$z$ & Intracellular 1,3-propanediol concentration in the reactor $\left(\mathrm{mmol}^{-1}\right)$ \\
$D$ & Dilution rate $\left(\mathrm{h}^{-1}\right)$ \\
$\mu$ & Specific growth rate $\left(\mathrm{h}^{-1}\right)$ \\
$\mu_{\mathrm{max}}$ & Maximum specific growth rate $\left(\mathrm{h}^{-1}\right)$ \\
$K$ & Monod saturation constant for the substrate $\left(\mathrm{mmol} \mathrm{l}^{-1}\right)$ \\
$c$ & Maximum residual substrate concentration $\left(\mathrm{mmol}^{-1}\right)$ \\
$d$ & Maximum product concentration $\left(\mathrm{mmol} \mathrm{l}^{-1}\right)$ \\
$a_{0}$ & Glycerol concentration in feed medium $\left(\mathrm{mmol} \mathrm{l}^{-1}\right)$ \\
$n_{\mathrm{s}}$ & Maintenance term of the substrate $\left(\mathrm{mmol} \mathrm{g}^{-1} \mathrm{~h}^{-1}\right)$ \\
$n_{\mathrm{p}}$ & Product formation under substrate-limited Condition $\left(\mathrm{mmolg}^{-1} \mathrm{~h}^{-1}\right)$ \\
$Y_{\mathrm{s}}$ & Maximum growth yield $(\mathrm{mmol} \mathrm{g})$ \\
$Y_{\mathrm{p}}$ & Maximum product yield $(\mathrm{mmol} \mathrm{g})$ \\
\hline \hline
\end{tabular}

where $c$ and $d$ are the critical values of the concentration of the substrate and product, respectively. The definitions of $\mu_{\max }$ and $K$ are also given in Table I. Clearly, if $y=c$ or $z=d$, then $\mu=0$. That is to say, the microorganism will stop growing when the concentration of the substrate or product reaches the critical value.

It is found from Refs. 11 and 12 that the substrate concentration has a great influence on the microbial growth rate. The microorganism grows very slowly not only at a low substrate concentration but also at a high substrate concentration. If the substrate is added with a steady dilution rate, where $D$ is constant, the concentration of the substrate will be excessively high. The excess substrate will limit the growth of the microorganism. A feasible method towards the problem is to add the substrate periodically. Due to this reason, we consider the periodically forced dilution rate in system (1), i.e.,

$$
D(t)=r(1+\varepsilon \sin (2 \pi t))
$$

In this paper, we study the microbial continuous culture model with the restrained growth rate (2) and periodically forced dilution rate (3). Notice that the forced system is nonautonomous, which makes plenty of results on the autonomous system no longer available. In recent years, few pioneering works have been done with periodically forced systems, such as a predator-prey model with seasonal prey harvesting, ${ }^{13}$ NF-kB oscillations with a circadian oscillation $^{14}$ and a seasonally forced predator-prey system with generalized Holling type IV functional response. ${ }^{15}$ These studies all focus on the physical model or population dynamics, and the dimension of these models is two. Here, we study the microbial continuous culture model with the periodically forced dilution rate, and the dimension is three, which is novel and more intricate.

The rest of this paper is organized as follows. In Sec. II, we discuss the equilibria of the system. In Sec. III, we analyse the saddle-node bifurcation, Hopf bifurcation, and its direction. In Sec. IV, we investigate the periodically forced system and display some bifurcation diagrams. Section V is the discussion of the paper.

\section{EXISTENCE AND TYPES OF EQUILIBRIA}

From expression (2), we have $0<y<c$ and $0<z<d$ for $\mu>0$. Following this, we can get

$$
\begin{aligned}
& \frac{\partial \mu}{\partial y}=\mu_{\max } \frac{c K-y^{2}-2 y K}{c(y+K)^{2}}\left(1-\frac{z}{d}\right), \\
& \frac{\partial \mu}{\partial z}=\frac{y^{2}-c y}{y+K} \frac{\mu_{\max }}{c d} .
\end{aligned}
$$

Let $y_{0}=\sqrt{K^{2}+K c}-K$, where $y_{0}$ is the result from imposing $\partial \mu / \partial y=0$. Then, we obtain the following results:

- If $y \in\left(0, y_{0}\right)$, then $\frac{\partial \mu}{\partial y}>0$. The growth rate of the microorganism monotonically increases with $y$. If $y \in\left(y_{0}, c\right)$, then $\frac{\partial \mu}{\partial y}<0$. The growth rate is restrained and monotonically decreases with $y$.

- $\frac{\partial \mu}{\partial z}<0$ for all $z \in(0, d)$. This means that the growth rate will decrease with the increase in the concentration of 1,3PD.

To verify the above results, one can see the trend of growth rate $\mu$ from Fig. 1 . It is easy to find that system (1) has a trivial equilibrium $E_{0}\left(0, a_{0}, 0\right)$. For any nontrivial equilibrium $E(x, y, z)$, it satisfies

$$
\left\{\begin{array}{l}
x(\mu-D)=0 \\
D\left(a_{0}-y\right)-x\left(n_{s}+\frac{\mu}{Y_{s}}\right)=0 \\
x\left(n_{p}+Y_{p} \mu\right)-D z=0 .
\end{array}\right.
$$

To get the solution of (4), we should solve a cubic polynomial equation. It is obvious from (4) that all equilibria lie on the line

$$
L_{0}:\left\{\begin{array}{l}
z=\left(a_{0}-y\right) \frac{n_{p}+Y_{p} D}{n_{s}+\frac{D}{Y_{s}}}, \\
x=\left(a_{0}-y\right) \frac{Y_{s} D}{Y_{s} n_{s}+D} .
\end{array}\right.
$$

Noticing that $x>0, z>0$, then all equilibria of system (1) should be in the region

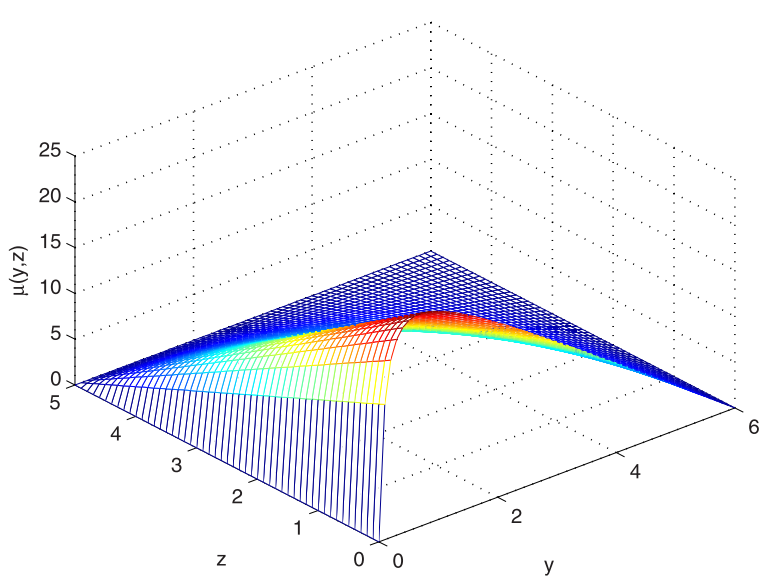

FIG. 1. The diagram of the function $\mu(y, z)$ for the case $\mu_{\max }=30, c=6$, $d=5$, and $K=0.1$. 


$$
R_{0}=\left\{(x, y, z) \in R^{3} \mid x>0,0<y<a_{0}, 0<z<d\right\} .
$$

Thus, we consider orbits of the model in $R_{0}$.

Substituting (5) in the first equation of (4), we obtain a cubic polynomial function with $y$

$$
F(y):=y^{3}+\omega_{2} y^{2}+\omega_{1} y+\omega_{0},
$$

where

$$
\begin{aligned}
& \omega_{0}= \frac{D c d K\left(n_{s}+\frac{D}{Y_{s}}\right)}{\mu_{m}\left(n_{p}+Y_{p} D\right)}, \\
& \omega_{1}= c a_{0}+\frac{D c d\left(n_{s}+\frac{D}{Y_{s}}\right)}{\mu_{m}\left(n_{p}+Y_{p} D\right)}-\frac{c d\left(n_{s}+\frac{D}{Y_{s}}\right)}{n_{p}+Y_{p} D}, \\
& \omega_{2}=d\left(\frac{n_{s}+\frac{D}{Y_{s}}}{n_{p}+Y_{p} D}-c-a_{0}\right) .
\end{aligned}
$$

Clearly, $\omega_{0}>0$. The number of equilibria is determined by the number of real roots of $F(y)=0$ in the interval $I_{0}$ $:=\left(0, a_{0}\right)$. Derivative

$$
F^{\prime}(y)=3 y^{2}+2 \omega_{2} y+\omega_{1} .
$$

Denote $\Delta$ the discriminant of $F^{\prime}(y)=0$ with $y$, then $\Delta=4 \omega_{2}^{2}-12 \omega_{1}$. If $\Delta \geq 0$, we know that $F^{\prime}(y)=0$ has two roots

$$
\xi_{ \pm}=\frac{-2 \omega_{2} \pm \sqrt{\Delta}}{6} .
$$

Then, we get

Lemma 1. System (1) has one trivial equilibrium $E_{0}(0$, $\left.a_{0}, 0\right)$ and at most two nontrivial equilibria in $R_{0}$.

(i) System (1) has two nontrivial equilibria in $R_{O}$ if and only if $\Delta>0,0<\xi_{+}<a_{0}, F\left(\xi_{+}\right)<0$, and $F\left(a_{0}\right)>0$;

(ii) System (1) has only one nontrivial equilibrium in $R_{0}$ if $\Delta>0,0<\xi_{+}<a_{0}$, and $F\left(\xi_{+}\right)=0$, or $F\left(a_{0}\right)<0$.

Proof. If $y=a_{0}$, we know that the system has a trivial equilibrium $E_{0}$ from (5). Clearly, equation $F(y)=0$ has at most three real solutions because $\operatorname{deg}(F)=3$. Just suppose that equation $E(y)=0$ has three real solutions $y_{1}, y_{2}$, and $y_{3}$, without loss of generality, we assume $y_{1}<y_{2}<y_{3}$. Then, $F(y)$ can be written as

$$
F(y)=\left(y-y_{1}\right)\left(y-y_{2}\right)\left(y-y_{3}\right)
$$

and we have

$$
F(0)=\omega_{0}=-y_{1} y_{2} y_{3} .
$$

In view of $\omega_{0}>0$, we obtain that equation $F(y)=0$ has at least one negative root, let $y_{1}<0$, as shown in Fig. 2(a). Therefore, $F(y)=0$ has at most two positive real roots in $I_{0}$. In other words, system (1) has at most two nontrivial equilibria in region $R_{0}$.

If system (1) has two nontrivial equilibria in $R_{0}$, then $F(y)=0$ has two different real roots $y_{2}<y_{3}$ in $I_{0}$. That is to say, $\Delta>0$, one can also find $0<y_{2}<\xi_{+}<y_{3}<a_{0}$. According to the property of continuous function, we get $F\left(\xi_{+}\right)<0, F\left(a_{0}\right)>0$, just as the statement (i), see Fig. 2(c).

For statement (ii), if $F\left(a_{0}\right)<0$, then $F(y)=0$ must have a root in $I_{0}$ for $F(0)=\omega_{0}>0$. System (1) has only one nontrivial equilibrium in $R_{0}$, see Fig. 2(b).

If $\xi_{+}<a_{0}$ and $F\left(\xi_{+}\right)=0$, then $\xi_{+}=y_{2}=y_{3}$. Therefore, $F=0$ has the two same positive solutions. In other words, system (1) has only one equilibrium in $R_{0}$, as shown in Fig. 2(d).

Remark 1. We denote the nontrivial equilibrium as $E(\bar{x}, \bar{y}, \bar{z})$. Let $E_{1}$ and $E_{2}$ be the two different nontrivial equilibria whenever they exit, whose corresponding y component satisfies $0<y_{1}<y_{2}<a_{0}$. For simplicity, we denote $\mu_{y}=\frac{\partial \mu}{\partial y}$, $\mu_{z}=\frac{\partial \mu}{\partial z}, \bar{\mu}_{y}=\left.\frac{\partial \mu}{\partial y}\right|_{E(\bar{x}, \bar{y}, \bar{z})}, \bar{\mu}_{z}=\left.\frac{\partial \mu}{\partial z}\right|_{E(\bar{x}, \bar{y}, \bar{z})}$, and $\bar{\mu}=\mu(\bar{x}, \bar{y}, \bar{z})$.

Theorem 1. For system (1), the trivial equilibrium $E_{0}(0$, $\left.a_{0}, 0\right)$ is

(i) a stable node if $\Upsilon<0$;

(ii) a hyperbolic saddle if $\Upsilon>0$;

(iii) a saddle-node if $\Upsilon=0$; where $\Upsilon=\frac{\mu_{\max } a_{0}}{k+a_{0}}\left(1-\frac{a_{0}}{c}\right)-D$.

Proof. For the trivial equilibrium $E_{0}$, we know $x=0$ is an invariant of the system, and the trivial steady state resides on this plane. Plugging $x=0$ into the last two equation of system (1), we obtain a linear system with the corresponding eigenvalues $\lambda_{1}=\lambda_{2}=-D$. From the first equation of system (1), we find that the third eigenvalue is

$$
\lambda_{3}=\Upsilon=\frac{\mu_{\max } a_{0}}{k+a_{0}}\left(1-\frac{a_{0}}{c}\right)-D .
$$

If $\Upsilon<0, E_{0}$ is an attracting node because it has three negative eigenvalues. If $\Upsilon>0, E_{0}$ is a hyperbolic saddle. If $\Upsilon=0$, then $-D,-D$, and 0 are the three eigenvalues of the system at $E_{0}$. Therefore, $E_{0}$ is a saddle-node and we need to calculate the one dimensional center manifold.

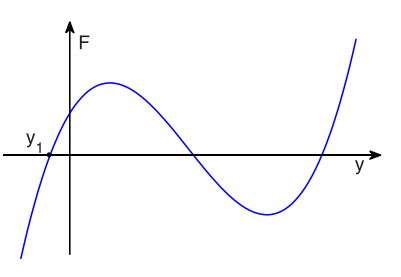

(a)

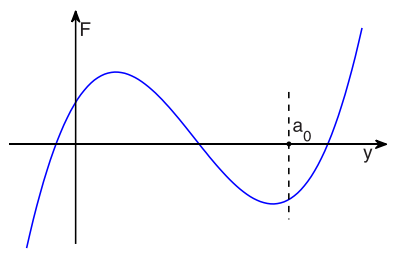

(b)

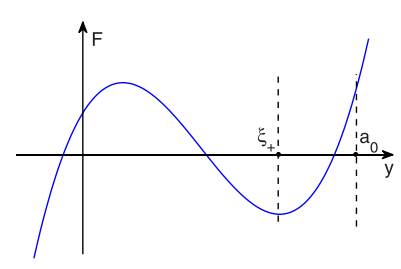

(c)

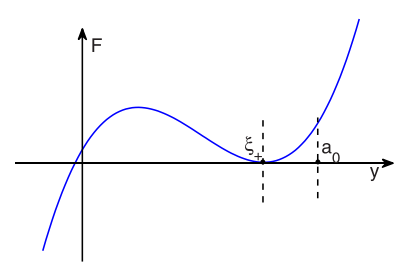

(d)

FIG. 2. Zeros of $F$. 
To obtain the one dimensional center manifold, some necessary transformation is done by us. We consider a polynomial system which has $y+K>0$ the same orbits as system (1). By a new time transformation $d t=(y+K) d \tau$ system (1) is topologically equivalent to

$$
\left\{\begin{array}{c}
\dot{x}=x\left[\frac{\mu_{\max }}{c d}(c-y)(d-z) y-D(y+K)\right], \\
\dot{y}=D\left(a_{0}-y\right)(y+K)-x\left[\left(n_{s}(y+K)\right.\right. \\
\left.\left.+\frac{\mu_{\max }}{Y_{s} c d}(c-y)(d-z) y\right)\right], \\
\dot{z}=x\left[n_{p}(y+K)+Y_{p} \frac{\mu_{\max }}{c d}(c-y)(d-z) y\right] \\
-D z(y+K) .
\end{array}\right.
$$

Instead of discussing the original system, we study the system (9) in this part. By the straightforward calculation, one can find that $\bar{V}_{1}, \bar{V}_{2}, \bar{V}_{3}$ are three eigenvectors of system (9)

$$
\bar{V}_{1}=\left(\begin{array}{c}
1 \\
\frac{\Phi}{D\left(K+a_{0}\right)} \\
\frac{\Psi}{D\left(K+a_{0}\right)}
\end{array}\right), \quad \bar{V}_{2}=\left(\begin{array}{l}
0 \\
1 \\
0
\end{array}\right), \quad \bar{V}_{3}=\left(\begin{array}{l}
0 \\
0 \\
1
\end{array}\right),
$$

where

$$
\begin{gathered}
\Phi=-K n_{s}-\left(n_{s}+\frac{\mu_{\max }}{Y_{s}}\right) a_{0}+\frac{\mu_{\max }}{c Y_{s}} a_{0}^{2}, \\
\Psi=K n_{p}+\left(n_{p}+Y_{p} \mu_{\max }\right) a_{0}-\frac{Y_{p}}{c} \mu_{\max } a_{0}^{2} .
\end{gathered}
$$

We derive the normal form on the one dimensional center manifold as follows. The translation

$$
\tilde{x}=x-0, \quad \tilde{y}=y-a_{0}, \quad \tilde{z}=z-0,
$$

can bring $E_{0}$ to the origin. Let

$$
\left(\begin{array}{c}
\tilde{x} \\
\tilde{y} \\
\tilde{z}
\end{array}\right)=T\left(\begin{array}{c}
X \\
Y \\
Z
\end{array}\right), \quad T=\left[\bar{V}_{1}, \bar{V}_{2}, \bar{V}_{3}\right] .
$$

Under the transformation $T$ which diagonalizes the Jacobian $J\left(E_{0}\right)$, system (9) becomes

$$
\left\{\begin{aligned}
\dot{X}= & l_{200} X^{2}+l_{110} X Y+l_{101} X Z+o\left(|X, Y, Z|^{2}\right), \\
\dot{Y}= & -D\left(K+a_{0}\right) Y+m_{200} X^{2}+m_{110} X Y \\
& +m_{101} X Z+o\left(|X, Y, Z|^{2}\right) \\
\dot{Z}= & -D\left(K+a_{0}\right) Z+n_{200} X^{2}+n_{110} X Y \\
& +n_{101} X Z+n_{011} Y Z+o\left(|X, Y, Z|^{2}\right)
\end{aligned}\right.
$$

where

$$
\begin{aligned}
l_{200}= & \left(\mu_{\max }-D-\frac{2}{c} \mu_{\max } a_{0}\right) \frac{\Phi}{D\left(K+a_{0}\right)} \\
& +\frac{\Psi \mu_{\max } a_{0}\left(a_{0}-c\right)}{D c d\left(K+a_{0}\right)}, \\
m_{200}= & \frac{-\Psi}{D\left(K+a_{0}\right)}\left[\frac{\mu_{\max } a_{0}\left(a_{0}-c\right)}{Y_{s} c d}+\left(n_{s}+\frac{c \mu_{\max }-2 \mu_{\max } a_{0}}{c Y_{s}}\right)\right. \\
& \left.+\frac{\Phi}{K+a_{0}}\right], \\
n_{200}= & \left(n_{p}+Y_{p} \mu_{\max }-\frac{2}{c} Y_{p} a_{0}\right) \frac{\Phi}{D\left(K+a_{0}\right)}-\frac{\Phi \Psi}{D\left(K+a_{0}\right)^{2}} \\
& +\frac{\Psi Y_{p} \mu_{\max } a_{0}\left(a_{0}-c\right)}{D c d\left(K+a_{0}\right)} .
\end{aligned}
$$

Here, we do not present other $l_{i j k}, m_{i j k}$, and $n_{i j k}$. In the following, we give the form of one dimensional center manifold. For $X \sim 0$, there exist a center manifold

$$
Y=\frac{m_{200}}{D\left(K+a_{0}\right)} X^{2}+o\left(X^{2}\right), \quad Z=\frac{n_{200}}{D\left(K+a_{0}\right)} X^{2}+o\left(X^{2}\right) .
$$

System (9) reduced on the one dimensional center manifold (10) is given by

$$
\dot{X}=l_{200} X^{2}+o\left(X^{2}\right) .
$$

Hence, if $l_{200} \neq 0, E_{0}$ is a saddle-node of codimension 1.

Theorem 2. For system (1), let $E(\bar{x}, \bar{y}, \bar{z})$ be a nontrivial equilibrium and $\Gamma(\bar{y}) \neq 0$, then $E$ is a hyperbolic saddle if $\Theta(\bar{y})<0$, it is anti-saddle if $\Theta(\bar{y})>0$, where

$$
\begin{aligned}
\Gamma(\bar{y}) & =D+\frac{\bar{x} \mu_{y}}{Y_{s}}-\bar{x} Y_{p} \mu_{z}, \\
\Theta(\bar{y}) & =\frac{\bar{x} \mu_{y}\left(n_{s} Y_{s}+D\right)}{Y_{s}}-\left(n_{p}+D Y_{p}\right) \bar{x} \mu_{z} .
\end{aligned}
$$

Proof. The Jacobian at E of system (1) is given by

$$
J(E)=\left[\begin{array}{ccc}
0 & \bar{x} \mu_{y} & \bar{x} \mu_{z} \\
-\left(n_{s}+\frac{D}{Y_{s}}\right) & -D-\frac{\bar{x}}{Y_{s}} \mu_{y} & -\frac{\bar{x}}{Y_{s}} \mu_{z} \\
n_{p}+D Y_{p} & \bar{x} Y_{p} \mu_{y} & \bar{x} Y_{p} \mu_{z}-D
\end{array}\right] .
$$

Then, we get the characteristic matrix

$$
A(E)=\left[\begin{array}{ccc}
\lambda & -\bar{x} \mu_{y} & -\bar{x} \mu_{z} \\
\frac{\lambda}{Y_{s}}+\left(n_{s}+\frac{D}{Y_{s}}\right) & \lambda+D & 0 \\
-Y_{p} \lambda-n_{p}-D Y_{p} & 0 & \lambda+D
\end{array}\right] .
$$

By the straightforward calculation, the characteristic equation of $A(E)$ has the form

$$
(\lambda+D)\left(\lambda^{2}+\Gamma(\bar{y}) \lambda+\Theta(\bar{y})\right)=0 .
$$

One can verify $\lambda=-D$ is always an eigenvalue, the other two eigenvalues of $J(E)$ are roots of 


$$
\lambda^{2}+\Gamma(\bar{y}) \lambda+\Theta(\bar{y})=0 .
$$

Hence, $E$ is a hyperbolic saddle if $\Theta(\bar{y})<0$ and $E$ is an antisaddle if $\Theta(\bar{y})>0$.

\section{BIFURCATIONS OF UNFORCED SYSTEM}

\section{A. Bifurcations analysis}

We study saddle-node bifurcation and Hopf bifurcation of the nontrivial equilibria in this section.

Theorem 3. Assume $\Gamma(\bar{y}) \neq 0$. If $F(\bar{y})=F^{\prime}(\bar{y})=0$, $\Theta(\bar{y})=0$, and $\tilde{l}_{200} \neq 0$, the nontrivial equilibrium of multiplicity 2 is a saddle-node of codimension 1, and system (1) undergoes a saddle-node bifurcation, where

$\tilde{l}_{200}=\left(\frac{n_{s}}{D}+\frac{1}{Y_{s}}\right)\left(D+n_{p}+D Y_{p}-Y_{p} n_{p}-\frac{n_{p}^{2}}{D}+\frac{Y_{s} n_{s}+D}{Y_{s}^{2}}\right)$.

Proof. If $F(\bar{y})=F^{\prime}(\bar{y})=0$, from (ii) of lemma1, two nontrivial equilibria $E_{2}$ and $E_{3}$ coalesce at one point $E$. That is to say, system (1) has one positive equilibrium.

We still study the system (9) in the following. If $\Theta(\bar{y})=0$, from (11) one can find that $0,-\Gamma(\bar{y})$, and $-D$ are the three eigenvalues of $J(E)$ with associated eigenvectors $\bar{V}_{1}, \bar{V}_{2}$, and $\bar{V}_{3}$, where

$$
\begin{aligned}
& \bar{V}_{1}=\left(\begin{array}{c}
1 \\
-\frac{n_{s}}{D}-\frac{1}{Y_{s}} \\
\frac{n_{p}}{D}+Y_{p}
\end{array}\right), \quad \bar{V}_{2}=\left(\begin{array}{c}
1 \\
\frac{n_{s}}{\Gamma(\bar{y})-D}-\frac{1}{Y_{s}} \\
Y_{p}+\frac{n_{p}}{\Gamma(\bar{y})-D}
\end{array}\right), \\
& \bar{V}_{3}=\left(\begin{array}{c}
0 \\
1 \\
-\frac{\mu_{y}}{\mu_{z}}
\end{array}\right) .
\end{aligned}
$$

By using translation $\hat{x}=x-\bar{x}, \hat{y}=y-\bar{y}$, and $\hat{z}=z-\bar{z}$, we can bring $E$ to the origin. Let

$$
\left(\begin{array}{c}
\hat{x} \\
\hat{y} \\
\hat{z}
\end{array}\right)=\bar{T}\left(\begin{array}{c}
X \\
Y \\
Z
\end{array}\right), \quad \bar{T}=\left[\bar{V}_{1}, \bar{V}_{2}, \bar{V}_{3}\right] .
$$

Under the translation $\bar{T}$, we diagonalize the linear part of system (9) and it becomes

$$
\left\{\begin{array}{l}
\dot{X}=\tilde{l}_{200} X^{2}+\tilde{l}_{110} X Y+\tilde{l}_{101} X Z+\tilde{l}_{011} Y Z+o\left(|X, Y, Z|^{2}\right), \\
\dot{Y}=-\Gamma(\bar{y}) Y+\sum_{i, j, k \in N}^{i+j+k=2} \tilde{m}_{i j k} X^{i} Y^{j} Z^{k}+o\left(|X, Y, Z|^{2}\right), \\
\dot{Z}=-D Z+\sum_{i, j, k \in N}^{i+j+k=2} \tilde{n}_{i j k} X^{i} Y^{j} Z^{k}+o\left(|X, Y, Z|^{2}\right) .
\end{array}\right.
$$

Here, we choose not to present the form of $\tilde{l}_{i j k}, \tilde{m}_{i j k}$, and $\tilde{n}_{i j k}$. For $X \sim 0$, there exists a center manifold

$$
Y=\frac{\bar{m}_{200}}{\Gamma(\bar{y})} X^{2}+o\left(X^{2}\right), \quad Z=\frac{\bar{n}_{200}}{D} X^{2}+o\left(X^{2}\right)
$$

System (1) reduced on the one dimension center manifold (12) is given by

$$
\dot{X}=\tilde{l}_{200} X^{2}+o\left(X^{2}\right) .
$$

Clearly, if $F(\bar{y})=F^{\prime}(\bar{y})=0$ and $\tilde{l}_{200} \neq 0$, immediately we know that the system on the center manifold is topologically equivalent to

$$
\dot{X}=\sigma X^{2}+o\left(X^{2}\right),
$$

so $E$ is a saddle-node of codimension 1 .

Theorem 4. A Hopf bifurcation occurs at $E(\bar{x}, \bar{y}, \bar{z})$, if $F(\bar{y})=0, \Gamma(\bar{y})=0$, and $\Theta(\bar{y})>0$.

Proof. From (11), one can find that the eigenvalues of $J(E)$ of system (9) have the form

$$
\lambda_{1}=-D, \quad \lambda_{2,3}=-\frac{\Gamma(\bar{y})}{2} \pm \frac{\sqrt{\Gamma^{2}(\bar{y})-4 \Theta(\bar{y})}}{2} .
$$

Following, we try to verify the transversality condition. Let $\chi=-\frac{\Gamma(\bar{y})}{2}$ be the real part of the complex eigenvalues of the characteristic equation.

Consider $D$ as the bifurcation parameter and fix all the other parameters, then suppose there exists $D=D_{0}$ such that

$$
\chi=\frac{\bar{x}\left(Y_{p} \bar{\mu}_{z}-\frac{\bar{\mu}_{y}}{Y_{s}}\right)-D_{0}}{2}=0, \quad \Theta\left(\bar{y}\left(D_{0}\right)\right)>0 .
$$

Therefore, system (1) has the eigenvalues

$$
\lambda_{2,3}= \pm i \sqrt{\Theta\left(\bar{y}\left(D_{0}\right)\right)},
$$

where $i$ represents an imaginary unit. The transversality condition can be verified as

$$
\frac{d \chi}{d D}=-\frac{1}{2} \Gamma^{\prime}\left(\bar{y}\left(D_{0}\right)\right)=-\frac{1}{2} \neq 0 .
$$

Hence, system (9) undergoes Hopf bifurcation.

In order to consider the stability and direction of bifurcating periodic solutions, we should compute the first Lyapunov coefficient $l_{1}$ of the Hopf Bifurcation.

We still study the topologically equivalent system (9). Without loss of generality, assume system (9) has a nontrivial equilibrium $E=(\bar{x}, \bar{y}, \bar{z}), \bar{\mu}_{y}$, and $\bar{\mu}_{z}$ have the same meaning as those in Remark 1.

The Jacobian at equilibrium $E(\bar{x}, \bar{y}, \bar{z})$ is given by

$$
J(E)=[J(1), J(2), J(3)],
$$

where 


$$
\begin{aligned}
& J(1)=\left(\begin{array}{c}
0 \\
-n_{s}(\bar{y}+K)+\frac{\bar{\mu}}{Y_{s}} \\
n_{p}(\bar{y}+K)+Y_{p} \bar{\mu}
\end{array}\right), \\
& \bar{x}\left(\bar{\mu}_{y}-D\right) \\
& J(2)=\left(\begin{array}{c}
\bar{x} \bar{y}_{y}-\bar{x} n_{s} \\
-D\left(2 \bar{y}+K-a_{0}\right)-\frac{\bar{x}}{Y_{s}} \bar{\mu}^{-} \bar{x}_{z} \\
-\frac{\bar{x}}{Y_{s}} \bar{\mu}_{z} \\
\bar{x} \bar{\mu}_{z} \\
-\frac{\bar{x}}{Y_{s}} \bar{\mu}_{z} \\
\bar{x} Y_{p} \bar{\mu}_{z}-D(\bar{y}+K)
\end{array}\right),
\end{aligned}
$$

One can verify that $\lambda=-D\left(K+a_{0}\right)$ is always an eigenvalue, and the other two eigenvalues of $J(E)$ are roots of

$$
\lambda^{2}-\mathcal{T}(\bar{y}) \lambda+\mathcal{S}(\bar{y})=0
$$

where

$$
\begin{aligned}
& \mathcal{T}=\bar{x} Y_{p} \bar{\mu}_{z}-\frac{\bar{x}}{Y_{s}}\left(\bar{\mu}_{y}-D\right)-D\left(K+a_{0}\right), \\
& \mathcal{S}=\bar{x}(\bar{y}+K)\left[\left(n_{s}+\frac{D}{Y_{s}}\right)\left(\bar{\mu}_{y}-D\right)-\bar{\mu}_{z}\left(n_{p}+Y_{p} D\right)\right] .
\end{aligned}
$$

To compute the first Lyapunov coefficient, we fix the parameter $D$ at its critical point $D_{0}$. Then, system (9) satisfies

$$
\mathcal{T}\left(\bar{y}\left(D_{0}\right)\right)=0, \quad \mathcal{S}\left(\bar{y}\left(D_{0}\right)\right)>0 .
$$

One can verify that $J(E)$ has three eigenvalues $\lambda_{1}=-D_{0}(K$ $\left.+a_{0}\right), \lambda_{2,3}= \pm i \sqrt{\mathcal{S}}$. Obviously, by using the following variable transformation

$$
\left\{\begin{array}{l}
\varepsilon_{1}=x-\bar{x} \\
\varepsilon_{2}=y-\bar{y} \\
\varepsilon_{3}=z-\bar{z} .
\end{array}\right.
$$

we can move the equilibrium to the origin. Therefore, system (9) becomes

$$
\left\{\begin{aligned}
\frac{d \varepsilon_{1}}{d \tau}= & A_{11} \varepsilon_{1}+A_{12} \varepsilon_{2}+A_{13} \varepsilon_{3}+B_{11} \varepsilon_{1} \varepsilon_{2}+B_{12} \varepsilon_{1} \varepsilon_{3} \\
& +B_{13} \varepsilon_{2} \varepsilon_{3}+B_{14} \varepsilon_{2}^{2}+C_{11} \varepsilon_{1} \varepsilon_{2}^{2}+C_{12} \varepsilon_{1} \varepsilon_{2} \varepsilon_{3} \\
& +C_{13} \varepsilon_{2}^{2} \varepsilon_{3}+D_{11} \varepsilon_{1} \varepsilon_{2}^{2} \varepsilon_{3} \\
\frac{d \varepsilon_{2}}{d \tau}= & A_{21} \varepsilon_{1}+A_{22} \varepsilon_{2}+A_{23} \varepsilon_{3}+B_{21} \varepsilon_{1} \varepsilon_{2}+B_{22} \varepsilon_{1} \varepsilon_{3} \\
& +B_{23} \varepsilon_{2} \varepsilon_{3}+B_{24} \varepsilon_{2}^{2}+C_{21} \varepsilon_{1} \varepsilon_{2}^{2}+C_{22} \varepsilon_{1} \varepsilon_{2} \varepsilon_{3} \\
& +C_{23} \varepsilon_{2}^{2} \varepsilon_{3}+D_{21} \varepsilon_{1} \varepsilon_{2}^{2} \varepsilon_{3} \\
\frac{d \varepsilon_{3}}{d \tau}= & A_{31} \varepsilon_{1}+A_{32} \varepsilon_{2}+A_{33} \varepsilon_{3}+B_{31} \varepsilon_{1} \varepsilon_{2}+B_{32} \varepsilon_{1} \varepsilon_{3} \\
& +B_{33} \varepsilon_{2} \varepsilon_{3}+B_{34} \varepsilon_{2}^{2}+C_{31} \varepsilon_{1} \varepsilon_{2}^{2}+C_{32} \varepsilon_{1} \varepsilon_{2} \varepsilon_{3} \\
& +C_{33} \varepsilon_{2}^{2} \varepsilon_{3}+D_{31} \varepsilon_{1} \varepsilon_{2}^{2} \varepsilon_{3}
\end{aligned}\right.
$$

where the coefficients of system (13) are given as follows:

$$
\begin{aligned}
& A_{11}=-D_{0} K+\left(\mu_{\max }-D_{0}\right) \bar{y}-\mu_{\max }\left(\frac{\bar{y}^{2}}{c}-\frac{\bar{y}^{2} \bar{z}}{c d}+\frac{\bar{y} \bar{z}}{d}\right), \quad A_{12}=\left(\mu_{\max }-D_{0}\right) \bar{x}-\mu_{\max }\left(\frac{2 \bar{x} \bar{y}}{c}-\frac{2 \bar{x} \bar{y} \bar{z}}{c d}+\frac{\bar{x} \bar{z}}{d}\right) \\
& A_{13}=\mu_{\max }\left(\frac{\bar{x} \bar{y}^{2}-c \bar{x} \bar{y}}{c d}\right), \quad B_{11}=\mu_{\max }\left(\frac{c-2 \bar{y}}{c}+\frac{2 \bar{y} \bar{z}}{c d}-\frac{\bar{z}}{d}\right)-D_{0}, \quad B_{12}=\mu_{\max }\left(\frac{\bar{y}^{2}}{c d}-\frac{\bar{y}}{d}\right), \quad B_{13}=\mu_{\max } \frac{2 \bar{x} \bar{y}}{c d}+\bar{x}\left(\mu_{\max }-D_{0}\right), \\
& B_{14}=\mu_{\max }\left(\frac{\bar{x} \bar{z}}{c d}-\frac{\bar{x}}{c}\right), \quad C_{11}=\mu_{\max }\left(\frac{\bar{z}}{c d}-\frac{1}{c}\right), \quad C_{12}=\mu_{\max }\left(\frac{2 \bar{y}}{c d}-\frac{1}{d}\right), \quad C_{13}=\frac{\mu_{\max }}{c d}, \quad D_{11}=\frac{\mu_{\max }}{c d}, \\
& A_{21}=-n_{s} K-\left(n_{s}+\frac{\mu_{\max }}{Y_{s}}\right) \bar{y}+\mu_{\max }\left(\frac{\bar{y}^{2}}{c Y_{s}}-\frac{\bar{z} \bar{y}^{2}}{c d Y_{s}}+\frac{\bar{y} \bar{z}}{d Y_{s}}\right), \quad A_{22}=D_{0}\left(a_{0}-K-2 \bar{y}\right)-\left(n_{s}+\frac{\mu_{\max }}{Y_{s}}\right) \bar{x} \\
& +\frac{\mu_{\max }}{Y_{s}}\left(\frac{2 \bar{x} \bar{y}}{c}-\frac{2 \bar{x} \bar{y} \bar{z}}{c d}+\frac{\bar{x} \bar{z}}{d}\right), \quad A_{23}=-\frac{\mu_{\max }}{Y_{s}}\left(\frac{\bar{x} \bar{y}^{2}}{c d}-\frac{\bar{x} \bar{y}}{d}\right), \quad B_{21}=-\left(n_{s}+\frac{\mu_{\max }}{Y_{s}}\right)+\frac{\mu_{\max }}{Y_{s}}\left(\frac{2 \bar{y}}{c}-\frac{2 \bar{x} \bar{y}}{c d}+\frac{\bar{z}}{d}\right), \\
& B_{22}=-\frac{\mu_{\max } \bar{y}}{Y_{s}}\left(\frac{\bar{y}}{c d}-\frac{1}{d}\right), \quad B_{23}=\frac{\mu_{\max }}{Y_{s}}\left(\frac{-2 \bar{y} \bar{z}}{c d}+\frac{\bar{x}}{d}\right), \quad B_{24}=-\frac{\mu_{\max }}{Y_{s}}\left(\frac{\bar{x} \bar{y}}{c d}-\frac{\bar{x}}{c}\right)-D_{0}, \quad C_{21}=-\frac{\mu_{\max }}{Y_{s}}\left(\frac{\bar{z}}{c d}-\frac{1}{c}\right), \\
& C_{22}=-\frac{\mu_{\max }}{Y_{s}}\left(\frac{2 \bar{y}}{c d}-\frac{1}{d}\right), \quad C_{23}=-\bar{x} \frac{\mu_{\max }}{c d Y_{s}}, \quad D_{21}=-\frac{\mu_{\max }}{c d Y_{s}}, \quad A_{31}=n_{p} K+\left(\mu_{\max } Y_{p}+n_{p}\right) \bar{y}-\mu_{\max } Y_{p}\left(\frac{\bar{y}^{2}}{c}-\frac{\bar{y}^{2} \bar{z}}{c d}+\frac{\bar{z} \bar{y}}{d}\right), \\
& A_{32}=-D_{0} \bar{z}+\left(\mu_{\max } Y_{p}+n_{p}\right) \bar{x}-\mu_{\max } Y_{p}\left(\frac{2 \bar{x} \bar{y}}{c}-\frac{2 \bar{x} \bar{y} \bar{z}}{c d}+\frac{\bar{x} \bar{z}}{d}\right), \quad A_{33}=-D_{0}(\bar{y}+K)+\mu_{\max } Y_{p}\left(\frac{\bar{x} \bar{y}^{2}}{c d}-\frac{\bar{x} \bar{y}}{d}\right), \\
& B_{31}=\mu_{\max } Y_{p}+n_{p}-\mu_{\max } Y_{p}\left(\frac{2 \bar{y}}{c}-\frac{2 \bar{z} \bar{y}}{c d}+\frac{\bar{z}}{d}\right), \quad B_{32}=\mu_{\max } Y_{p}\left(\frac{\bar{y}^{2}-c \bar{y}}{c d}\right), \quad B_{33}=\mu_{\max } Y_{p}\left(\frac{2 \bar{y} \bar{z}-c \bar{x}}{c d}\right)-D_{0} \\
& B_{34}=\mu_{\max } Y_{p}\left(\frac{\bar{x} \bar{z}}{c d}-\frac{\bar{x}}{c}\right), \quad C_{31}=\mu_{\max } Y_{p}\left(\frac{\bar{z}}{c d}-\frac{1}{c}\right), \quad C_{32}=\mu_{\max } Y_{p}\left(\frac{2 \bar{y}}{c d}-\frac{1}{d}\right), \quad C_{33}=\mu_{\max } Y_{p} \frac{\bar{x}}{c d}, \quad D_{31}=\frac{\mu_{\max } Y_{p}}{c d} .
\end{aligned}
$$

Then, we write system (9) in terms of multilinear functions $B$ and $C$

$$
\frac{d \varepsilon}{d \tau}=A \varepsilon+\frac{1}{2} B(\varepsilon, \varepsilon)+\frac{1}{6} C(\varepsilon, \varepsilon, \varepsilon)+o\left(\|\varepsilon\|^{3}\right),
$$


where $A=A\left(D_{0}\right)$, the multilinear functions $B$ and $C$ have the form

$$
B(\xi, \eta)=\left[\begin{array}{l}
B_{11} \rho_{1}+B_{12} \rho_{2}+B_{13} \rho_{3}+2 B_{14} \rho_{4} \\
B_{21} \rho_{1}+B_{22} \rho_{2}+B_{23} \rho_{3}+2 B_{24} \rho_{4} \\
B_{31} \rho_{1}+B_{32} \rho_{2}+B_{33} \rho_{3}+2 B_{34} \rho_{4}
\end{array}\right]
$$

and

$$
C(\xi, \eta, \zeta)=\left[\begin{array}{l}
2 C_{11} \rho_{5}+C_{12} \rho_{6}+2 C_{13} \rho_{7} \\
2 C_{21} \rho_{5}+C_{22} \rho_{6}+2 C_{23} \rho_{7} \\
2 C_{31} \rho_{5}+C_{32} \rho_{6}+2 C_{33} \rho_{7}
\end{array}\right] .
$$

For the planar vectors $\xi=\left(\xi_{1}, \xi_{2}\right)^{T}, \eta=\left(\eta_{1}, \eta_{2}\right)^{T}$, and $\zeta=\left(\zeta_{1}, \zeta_{2}\right)^{T}$, we have

$$
\begin{aligned}
& \rho_{1}=\xi_{1} \eta_{2}+\xi_{2} \eta_{1}, \quad \rho_{2}=\xi_{1} \eta_{3}+\xi_{3} \eta_{1}, \quad \rho_{3}=\xi_{2} \eta_{3}+\xi_{3} \eta_{2}, \\
& \rho_{4}=\xi_{2} \eta_{2}, \quad \rho_{5}=\xi_{1} \eta_{2} \zeta_{2}+\xi_{2} \eta_{1} \zeta_{2}+\xi_{2} \eta_{2} \zeta_{1}, \\
& \rho_{6}=\xi_{1} \eta_{2} \zeta_{3}+\xi_{1} \eta_{3} \zeta_{2}+\xi_{2} \eta_{1} \zeta_{3}+\xi_{2} \eta_{3} \zeta_{1}+\xi_{3} \eta_{1} \zeta_{2}+\xi_{3} \eta_{2} \zeta_{1}, \\
& \rho_{7}=\xi_{2} \eta_{2} \zeta_{3}+\xi_{2} \eta_{3} \zeta_{2}+\xi_{3} \eta_{2} \zeta_{2} .
\end{aligned}
$$

In the following, to obtain the first Lyapunov coefficient we should calculate the eigenvectors of matrix $A\left(D_{0}\right)$ for the eigenvalues $\lambda_{2,3}$ and the eigenvector of matrix $A^{T}\left(D_{0}\right)$. The matrix $A\left(D_{0}\right)$ has the form:

$$
A\left(D_{0}\right)=\left[\begin{array}{lll}
A_{11} & A_{12} & A_{13} \\
A_{21} & A_{22} & A_{23} \\
A_{31} & A_{32} & A_{33}
\end{array}\right] .
$$

For simplicity, denote $\omega=\sqrt{\mathcal{S}\left(\bar{y}\left(D_{0}\right)\right)}$ and $\lambda_{2,3}= \pm i \omega$. Let $q_{1}=(\alpha, \beta, \gamma)^{T} \in C^{3}$ be eigenvector of matrix $A\left(D_{0}\right)$. For $A q_{1}=i \omega q_{1}$, we have

$$
\left\{\begin{array}{l}
\alpha w i-\beta \bar{x}\left(\bar{\mu}_{y}-D_{0}\right)-\gamma \bar{x} \bar{\mu}_{z}=0 \\
\alpha\left[\frac{i \omega}{Y_{s}}+n_{s}(\bar{y}+K)+\frac{\bar{\mu}}{Y_{s}}\right]+\beta\left[i \omega+D_{0}(\bar{y}+K)\right]=0 \\
\alpha\left[-i \omega Y_{p}-n_{p}(\bar{y}+K)-\bar{\mu} Y_{p}\right]+\gamma\left[i \omega+D_{0}(\bar{y}+K)\right]=0
\end{array}\right.
$$

By solving (14), we can get

$$
q_{1}=\left(\begin{array}{c}
1 \\
-\frac{i \omega+\bar{\mu}+(\bar{y}+K) n_{s} Y_{s}}{Y_{s}[i \omega+D(\bar{y}+K)]} \\
\frac{i Y_{p} \omega+n_{p}(\bar{y}+K)+Y_{p} \bar{\mu}}{i \omega+D(\bar{y}+K)}
\end{array}\right)
$$

Let $\bar{q}, p \in C^{3}$. For $A \bar{q}=-i \omega \bar{q}$ and $A^{T} p=-i \omega p$, we have

$$
\bar{q}=\left(\begin{array}{c}
1 \\
\frac{-i \omega+\bar{\mu}+(\bar{y}+K) n_{s} Y_{s}}{i \omega-D_{0}(\bar{y}+K)} \\
\frac{i Y_{p} \omega-n_{p}(\bar{y}+K)-Y_{p} \bar{\mu}}{-i \omega+D_{0}(\bar{y}+K)}
\end{array}\right), \quad p=\left(\begin{array}{c}
1 \\
\frac{i \omega+D_{0}(\bar{y}+K)}{\bar{x}\left(\bar{\mu}-D_{0}\right)} \\
\frac{i \omega+D_{0}(\bar{y}+K)}{\bar{x} \bar{\mu}_{z}}
\end{array}\right) .
$$

Taking $p=p, q=\frac{q_{1}}{\left\langle p \cdot q_{1}\right\rangle}$ we achieve the necessary normalization $\langle p, q\rangle=1$. Then, we can compute the first Lyapunov coefficient of system (9) (Ref. 16)

$$
\begin{aligned}
l_{1}= & \frac{1}{2 \omega} \operatorname{Re}\left[\langle p, C(q, q, \bar{q})\rangle-2\left\langle p, B\left(q, A^{-1} B(q, \bar{q})\right)\right\rangle\right. \\
& \left.+\left\langle p, B\left(\bar{q},\left(2 i \omega I_{3}-A\right)^{-1} B(q, q)\right)\right\rangle\right] .
\end{aligned}
$$

Let $l_{n}(\mathrm{n} \in \mathrm{N})$ be the Lyapunov coefficients of the equilibrium $E(\bar{x}, \bar{y}, \bar{z})$. In order to prove the existence of a generic Hopf bifurcation, in addition to transversality condition, we also need to verify that the first Lyapunov coefficient $l_{1}$ $\neq 0$. Generally, we have the following three cases for system (9)

(1) If $l_{1}<0$, the system undergoes supercritical Hopf bifurcation;

(2) If $l_{1}>0$, the system undergoes subcritical Hopf bifurcation;

(3) If $l_{1}=0$, the system undergoes degenerate Hopf bifurcation. Moreover, Hopf bifurcation is of codimension 2 in a small neighborhood of BT singularities of codimension 3 in the parameter space. ${ }^{17,18}$

\section{B. Numerical simulations}

In this section, we select some parameter values to show the existence of the Hopf bifurcation and fold (saddle-node) bifurcation. Some phase portraits, bifurcation curves, bifurcation diagram, and time series of limit cycle are given in Figs. 3 and 4. All these figures are obtained by software package MATLAB and software package AUTO. ${ }^{19}$ For convenience, we fix $K=0.1, c=10, d=3, n_{s}=-0.1, Y_{s}=4$, and $\mu_{\text {max }}=30$ and select $n_{p}, Y_{p}, a_{0}$, and $D$ as free parameters.

Figure 3(b) shows the bifurcation diagram for $n_{p}=0.018$ and $Y_{p}=0.001$. On the solid (dashed) line, the equilibrium is stable (unstable), and the sign of $H(L)$ represents the Hopf (fold) bifurcation point. The saddle point and the other nontrivial equilibrium collide at the fold bifurcation point $(L)$ and then disappear when parameter $D$ crosses the vertical line $D=0.42$ to the left of it. Figure 3(c) shows the bifurcation curves for $n_{p}=0.018$ and $Y_{p}=0.001$; here, we select $a_{0}$ and $D$ as the bifurcation parameters. The blue line in the figure represents the Hopf bifurcation curve and the black line represents the fold bifurcation curve. By the computation of the AUTO package, we find that all the first Lyapunov coefficient of the Hopf points in Fig. 3(c) satisfies $l_{1}<0$, that is to say, system (1) undergoes supercritical Hopf bifurcation. When the Hopf bifurcation curve is crossed to the left part, a stable limit cycle appears and the stable equilibrium becomes unstable. Besides, phase portrait of a stable limit cycle which bifurcates from the supercritical Hopf point and corresponding time series are given in Figs. 4(a) and 4(b), respectively.

Figure 3(d) shows bifurcation curves for $n_{p}=0.026$ and $Y_{p}=0.004$; here, we do not present the corresponding bifurcation diagram because it has a similar shape to that in Fig. 3(b). The blue line denotes the Hopf bifurcation curve, and the black line denotes the fold bifurcation curve. The small triangle $\left(D=0.41\right.$ and $\left.a_{0}=6.1\right)$ in the middle of the Hopf bifurcation curve represents a degenerate Hopf bifurcation 
(a)

$\Delta$
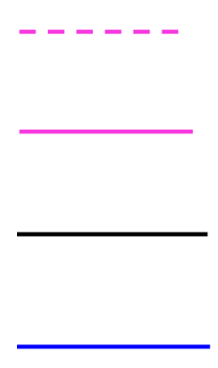

Degenerate Hopf point

Unstable solution

Stable solution

Fold bifurcation curve

Hopf bifurcation curve
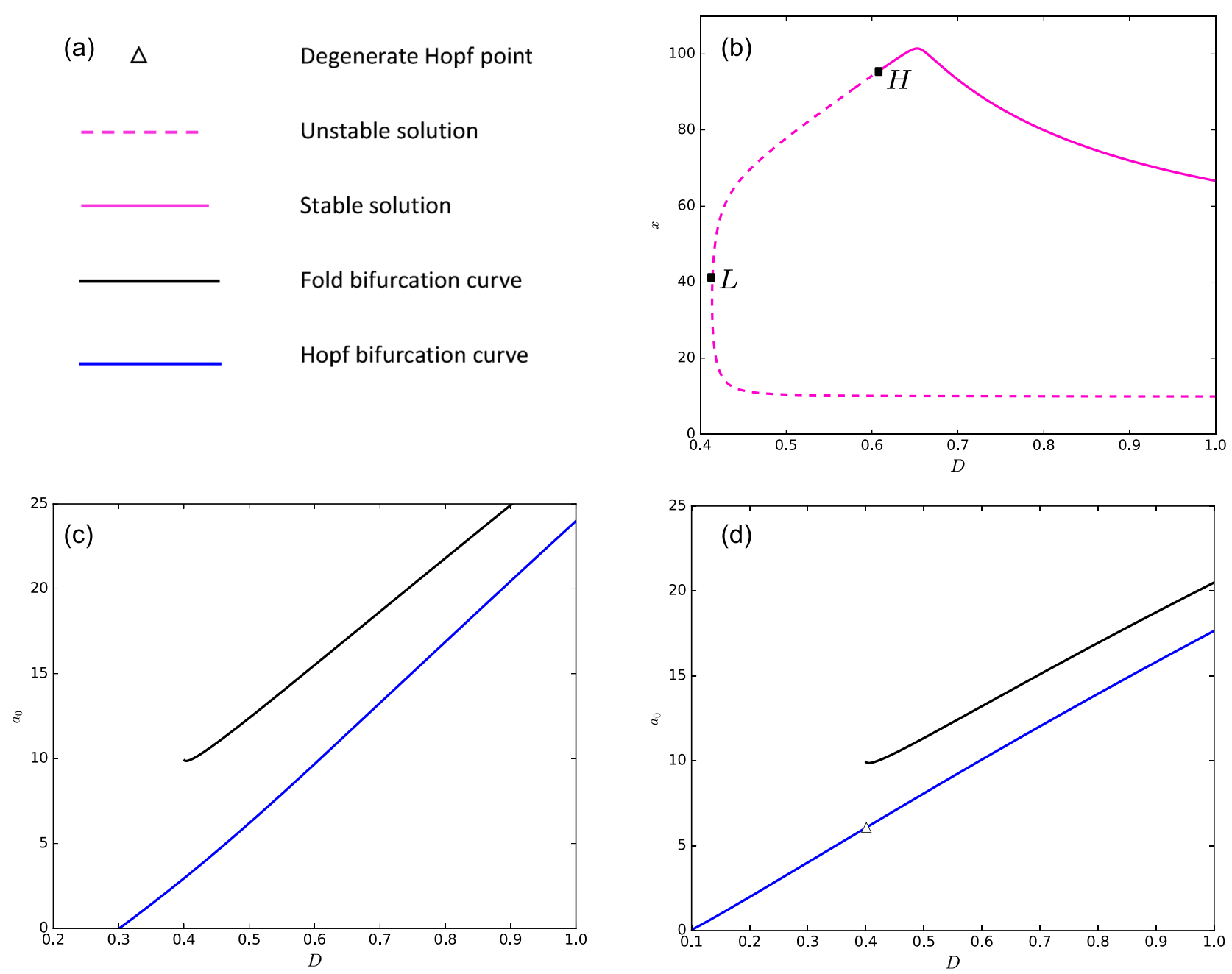

FIG. 3. (b) Bifurcation diagram for $n_{p}=0.018$ and $Y_{p}=0.001$. The solid (dashed) line represents the stable (unstable) equilibrium. The letter $\mathrm{H}$ (L) represents the Hopf (fold) bifurcation point. (c) The bifurcation curves for $n_{p}=0.018$ and $Y_{p}=0.001$. (d) The bifurcation curves for $n_{p}=0.026$ and $Y_{p}=0.004$. The blue (black) line of (c) and (d) represents the Hopf (fold) bifurcation curve, where the small triangle in the blue line of (d) represents a degenerate Hopf bifurcation point.

point, which means the first Lyapunov coefficient $l_{1}=0$ at this point. In addition, we can find that if $D>0.41(<0.41)$, then $l_{1}>0(<0)$, the system undergoes subcritical (supercritical) Hopf bifurcation. When the upper branch of the Hopf bifurcation curve $(D>0.41)$ is crossed to the right side, system (1) generates a stable equilibrium point and an unstable limit cycle appears. The phase portrait of the unstable limit cycle and time series are given in Figs. 4(c) and 4(d), respectively.

\section{PERIODICALLY FORCED SYSTEM}

\section{A. Bifurcations}

In this section, we try to investigate the dynamic behaviors of a periodically forced microbial continuous culture system. We use the software package AUTO to obtain the bifurcation diagram. The model can be expressed as

$$
\left\{\begin{array}{l}
\frac{d x}{d t}=x(\mu-D(t)), \\
\frac{d y}{d t}=D(t)\left(a_{0}-y\right)-x\left(n_{s}+\frac{\mu}{Y_{s}}\right), \\
\frac{d z}{d t}=x\left(n_{p}+Y_{p} \mu\right)-D(t) z .
\end{array}\right.
$$

where $\mu$ has the same expression as it in (2), and

$$
D(t)=r(1+\varepsilon \sin (2 \pi t)) .
$$

In this system, $r$ and $\varepsilon$ are added parameters. The timeperiodic function $D(t)$ describes the influence of periodic variability of the dilution on the dynamic behaviors. The time is scaled to make a period 1 in length. To make $D(t)>0$, we have $0<\varepsilon<1$. In AUTO package, the forced system can be done by adding a nonlinear oscillator with the desired periodic forcing as one of the solution components. In our case, the forced system can be transformed into the autonomous five dimensional system

$$
\left\{\begin{array}{l}
\frac{d x}{d t}=x(\mu-r(1+\varepsilon v)) \\
\frac{d y}{d t}=r(1+\varepsilon v)\left(a_{0}-y\right)-x\left(n_{s}+\frac{\mu}{Y_{s}}\right) \\
\frac{d z}{d t}=x\left(n_{p}+Y_{p} \mu\right)-r(1+\varepsilon v) z \\
\frac{d v}{d t}=v+2 \pi w-v\left(v^{2}+w^{2}\right) \\
\frac{d w}{d t}=w-2 \pi v-w\left(v^{2}+w^{2}\right)
\end{array}\right.
$$




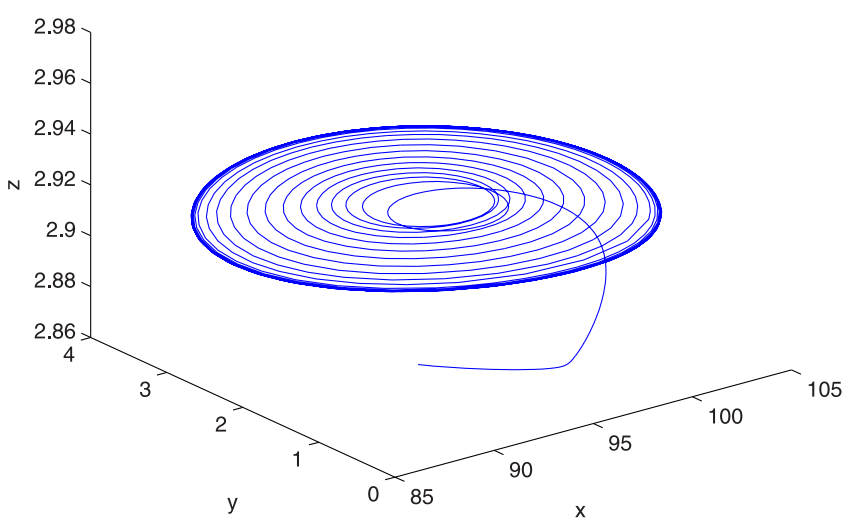

(a)

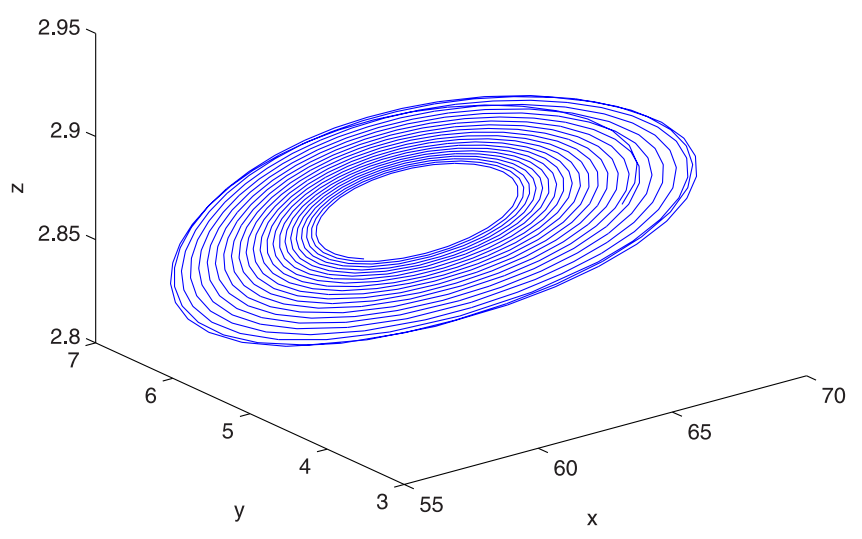

(c)
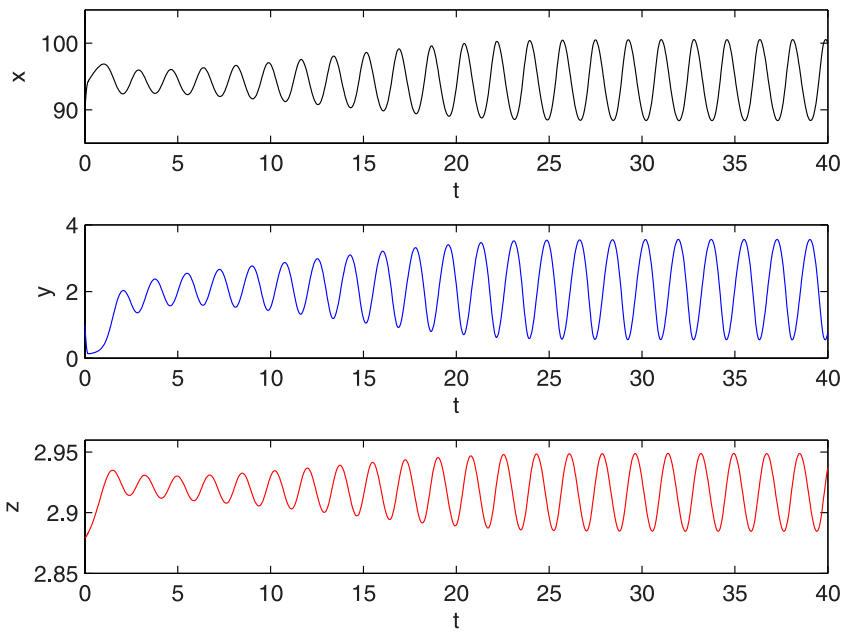

(b)
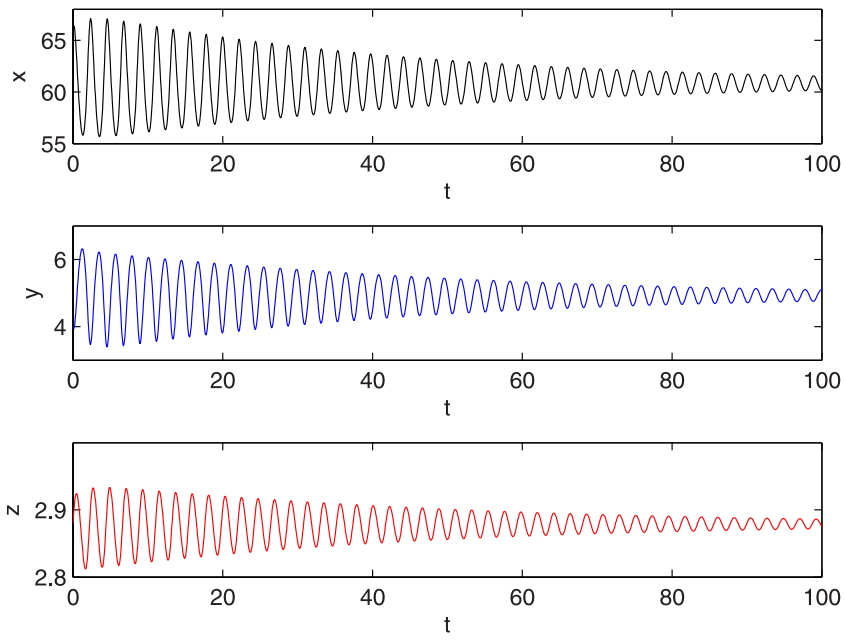

(d)

FIG. 4. Phase portrait of the limit cycle and corresponding time series. (a) A stable limit cycle generated by supercritical Hopf bifurcation for $n_{p}=0.018$, $Y_{p}=0.001$, and $D=0.6$. (b) Time series of the stable limit cycle. (c) An unstable limit cycle generated by subcritical Hopf bifurcation for $n_{p}=0.026$, $Y_{p}=0.004$, and $D=0.61$. (d) Time series of the unstable limit cycle.

and the last two equations of (16) have the asymptotically stable solutions $v=\sin (2 \pi t), w=\cos (2 \pi t)$. For $\varepsilon=0$, the system reduces to the autonomous system (1); if the unforced system has equilibrium $\left(x^{*}, y^{*}, z^{*}\right)$, then the periodically forced system has periodic solution $\left(x^{*}, y^{*}, z^{*}, \sin 2 \pi t\right.$, $\cos 2 \pi t)$. For $\varepsilon \neq 0$, we like to find if the periodic solution can survive and bifurcate. Parameter values for which the period $T_{h}$ of the appearing limit cycle is integer play an important role. Because in these cases, the ratio between the period $T_{h}$ and the period of forcing function is integer.

We use the Poincaré map to study the dynamics of the continuous five dimensional system. The first return map can be defined as

$$
\begin{gathered}
\mathcal{P}:(x(0), y(0), z(0), v(0), w(0)) \mapsto \\
(x(1), y(1), z(1), v(1), w(1)) .
\end{gathered}
$$

The stable (unstable) fixed points of the $k$ th iterate of the map correspond to the stable (unstable) periodic solutions with period $k$ of the forced system. We can refer these points to period $k$ fixed points. Moreover, closed and regular invariant curves of the Poincaré map correspond to quasiperiodic solutions (invariant tori), while irregular sets represent the chaotic solutions (strange attractors) of the five dimensional system. As parameters changed, the fixed point of the Poincare map of the forced system can bifurcate, and it will change its stability or disappear. For the bifurcation diagrams below, we use the following notations for codimension one bifurcation curves and codimension two bifurcation points of the map $\mathcal{P}$.

- $h^{(k)}$, Hopf (Neimark-Sacker) bifurcation curve. For parameter values on this curve, the map has a period $k$ fixed point with a pair of multipliers on the unit circle $\mu_{1,2}^{k}=e^{ \pm i \omega}, 0<\omega<\pi$.

- $f^{(k)}$, flip (Period doubling) bifurcation curve. For parameter values on this curve, the map has a period $k$ fixed point with a multiplier $\mu_{1}^{k}=-1$.

- $t^{(k)}$, fold (tangent) bifurcation curve. For parameter values on this curve, the map has a period $k$ fixed point with a multiplier $\mu_{1}^{k}=1$. 


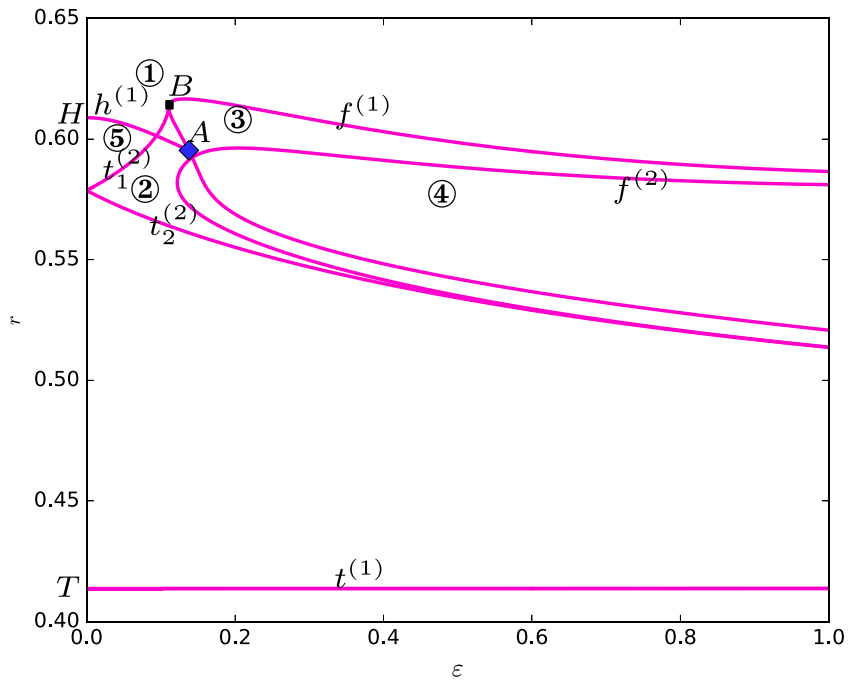

FIG. 5. Bifurcation diagram of the forced system for $n_{p}=0.018$ and $Y_{p}=0.001$. The solutions of the original continuous system are as follows: region 1-stable period-one solution, region 2-unstable period-one solution, stable and unstable period-two solutions, region 3-unstable period-one solution and stable period-two solutions, region 4-unstable period-one and period-two solutions, stable period-four solution or chaos in some subregion, and region 5-unstable period-one solution and stable quasiperiodic solution.

We have to mention that the description of the following diagrams is about the Poincaré map. To make the diagrams more readable, some necessary explanations for solutions of the five dimensional system in different regions are given in figure captions.

Figure 5 is the bifurcation diagram of the forced system in the $(\varepsilon, r)$-plane and the parameter values are selected as those in Fig. 3(c). At this group of parameter values, the unforced system has a stable limit cycle and its asymptotic period $T_{h}=1.72$. On the $r$-axis, point $H$ represents the Hopf bifurcation in the unforced system and it is the origin of curve $h^{(1)}$. Point $T$ represents the fold bifurcation in the unforced system and it is the origin of curve $t^{(1)}$.

Two fixed points of period-one collide on the curve $t^{(1)}$, forming a nonhyperbolic point, then disappear when $t^{(1)}$ is crossed to the below. Curve $h^{(1)}$ is formed by continuation of a Neimark-Sacker bifurcation of Poincaré map $\mathcal{P}$. As the parameter value of $r$ crosses curve $h^{(1)}$ from region 1 to region 5, stable fixed point of $\mathcal{P}$ changes its stability and a stable closed invariant curve appears. While continuing the curve $h^{(1)}$ from left to right on the $(\varepsilon, r)$-plane, both multipliers $\mu_{1,2}^{1}$ of the fixed point vary smoothly and become equal to -1 as the terminal point $A$ is reached. This is a codimension two bifurcation point called strong resonance $1: 2$, and the different types of resonances have been well studied in previous works. ${ }^{20,21}$ Thus, passing through the point $A$ there is a bifurcation curve related to period-two orbits. Curve $f^{(1)}$ is the flip bifurcation curve. Along this curve away from $A$, the fixed point has a simple multiplier $\mu_{1}^{1}=-1$. The two branches of curve $f^{(1)}$ can be obtained by numerical continuation starting from $A$ in two directions. Crossing curve $f^{(1)}$ from region 1 to region 3 above point $A$ results in the appearance of a couple of period-two fixed points, while the stable period-one fixed point becomes unstable. When $f^{(1)}$ is crossed from region 3 to region 2, another pair of unstable period-two fixed points appear and the period-one fixed point becomes a repelling point.

The analysis of the flip bifurcation on the upper branch of $f^{(1)}$ shows that there is another codimension two bifurcation point $B$ at which a nondegenerate generalized flip bifurcation occurs. Thus, crossing the part of $f^{(1)}$ located above the point $B$ leads to a pair of attracting period-two fixed points. Moreover, there is a tangent bifurcation curve $t_{1}^{(2)}$ whose root is point $B$. The tangent bifurcation curve has another branch $t_{2}^{(2)}$, and these two branches terminate at the same point on the $r$-axis. If the curve $t_{1}^{(2)}$ or $t_{2}^{(2)}$ is crossed from region 2, all the period-two fixed points disappear. If $f^{(2)}$ is crossed from region 3 to region 4, the two stable period-two fixed points lose its stability. Certainly, flip bifurcation curves $f^{(4)}, f^{(8)}, \ldots$, always exist, and this cascade of period doubling leads to strange attractors in some subregions of region 4 .

Figure 6 is the bifurcation diagram of the forced system in $(\varepsilon, r)$-plane, and the parameter values are fixed as those in Fig. 3(d). The asymptotic period of the unstable limit cycle of the unforced system is $T_{h}=2.22$. On the $r$-axis, the point $T$ corresponding to the fold bifurcation point in the unforced system is the root of curve $t^{(1)}$. In Fig. 6(a), curves $t^{(2)}$ and

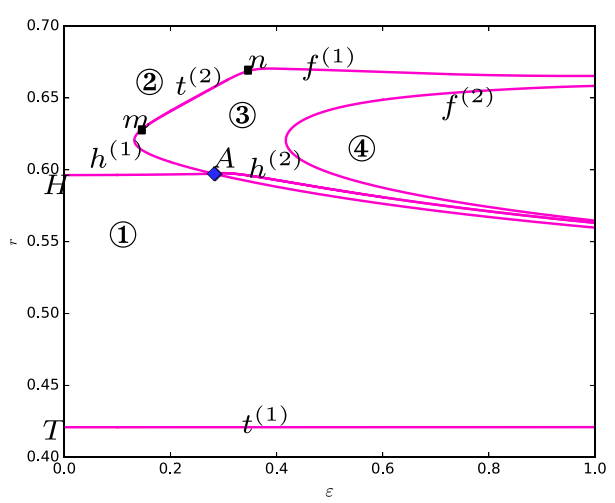

(a)

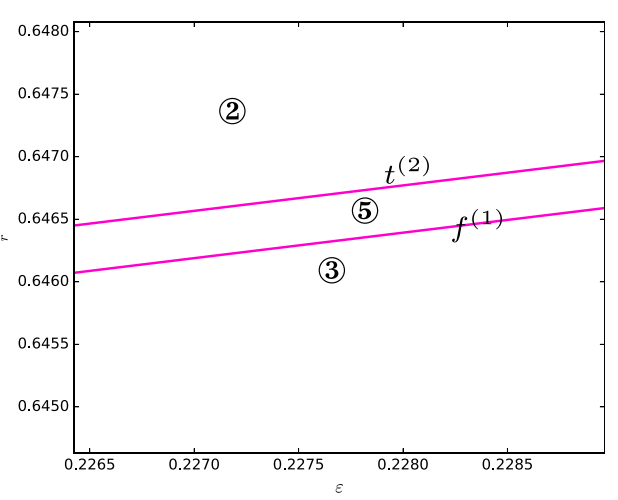

(b)

FIG. 6. The bifurcation diagram of the forced system for $n_{p}=0.026$ and $Y_{p}=0.004$. (a) Bifurcation diagram. (b) The partial enlargement drawing of (a) for the part $\mathrm{mn}$. Solutions of the original continuous system are as follows, region 1- stable and unstable period-one solution, region 2-stable period-one solution, and unstable quasiperiodic solution. region 3-unstable period-one and unstable period-two solutions, region 4- unstable period-one solution, unstable period-two solutions and period-four solutions or chaos in some subregion, and region 5-unstable period-one and unstable period-two solutions. 
$f^{(1)}$ have two crossover points $m$ and $n$, which are too close to distinguish so we present an enlargement as shown in Fig. 6(b). Point $H$ represents the subcritical Hopf bifurcation in the unforced system. It is the initial point of curve $h^{(1)}$ which terminates at point $A$, a 1:2 resonance, and point $A$ is the origin of the Neimark-Sacker bifurcation curve $h^{(2)}$. Moreover, there is a flip bifurcation curve $f^{(1)}$, at which a pair of period-two fixed points appear that pass through the point $A$.

In Fig. 6, if $t^{(1)}$ is crossed to the below, two period-one fixed points collide on $t^{(1)}$ and then disappear. If the curve $h^{(1)}$ is crossed from region 1 to region 2 , the unstable fixed point will turn into stable and an unstable closed invariant curve will appear. Two pairs of period-two fixed points appear when $t^{(2)}$ is crossed from region 2 to region 5 in (b). They are a pair of saddle fixed points and a pair of repelling fixed point. The saddles disappear when $f^{(1)}$ is crossed from region 5 to region 3 , while the two repelling fixed points will become attracting when crossing the curve $h^{(2)}$ to the below. The attracting fixed points will disappear when $f^{(1)}$ is crossed to region 1 . When $f^{(2)}$ is crossed from region 3 to region 4 , the two repelling period-two points become saddle fixed points and period-four solution appear.

\section{B. Solutions, chaos, and bistability}

In the periodically forced system (15), the equilibrium of system (1) becomes the periodic solution of period $T=1$ due to the adding nonlinear oscillator with frequency $\omega=2 \pi$. Since there are no equilibrium solutions of system (15), the bifurcation types are those of periodic orbits. Periodic forcing is a key feature in the model, and it can induce different bifurcations. We find fold bifurcations of periodic orbits, period-doubling bifurcations, and torus bifurcations, which can lead to the appearance of various solutions, see Figs. 7 and 8.

In this section, the parameter values are the same as those in Sec. IV, where $n_{p}, Y_{p}, r$, and $\varepsilon$ are selected as free parameters. Figure 7 (a) is a stable period-two orbit for $n_{p}=0.026, Y_{p}=0.004, r=0.61$, and $\varepsilon=0.17$; one can find that the period of the solution is two from the time series. In fact, period-two orbits can be found in many regions of Figs. 5 and 6 (region 2, region 3, and region 4). When perioddoubling bifurcation of period-two orbit occurs, period-two orbit changes the stability and period-four orbit appears. Figure $7(\mathrm{c})$ is a stable period-four orbit for $n_{p}=0.018$, $Y_{p}=0.001, r=0.594$, and $\varepsilon=0.2$, and from Fig. $7(\mathrm{~d})$ we know that the period is $T=4$. Period-eight orbits also exist in some regions of Figs. 5 and 6 as a result of bifurcation of period-four orbit; here, we do not show it. Figure 7(e) shows a stable torus for $n_{p}=0.018, Y_{p}=0.001, r=0.595$, and $\varepsilon=0.08$, which arises from a torus bifurcation. We cannot judge the period of the torus from the corresponding time series because it has an infinite period. Thus, we give the Poincaré section of the torus, see Fig. 7(g). The closed curve indicates that the phase portrait of Fig. 7(e) is torus, not chaos. Moreover, some other torus can be found in region 5 of Fig. 5 and region 2 of Fig. 6.

Following, we discuss the chaotic attractor in periodically forced system (15). To verify the existence of chaos, we show the spectrum of the Largest Lyapunov exponents of system (15), see Figs. 8(a) and 8(b). It is calculated based on the algorithm in Ref. 22. When the largest Lyapunov exponent of the time series $\lambda>0$, the corresponding attractor can be regarded as the chaotic attractor. Figure 8(a) is the spectrum of the Largest Lyapunov exponents in the $(\lambda-\varepsilon)$ plane (a)

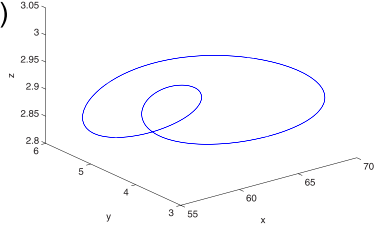

(b)

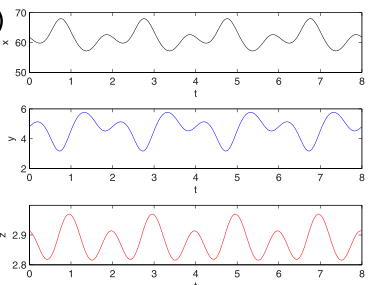

(c) ${ }^{3}$

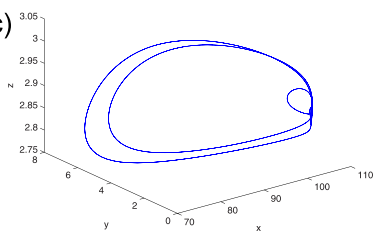

(d)

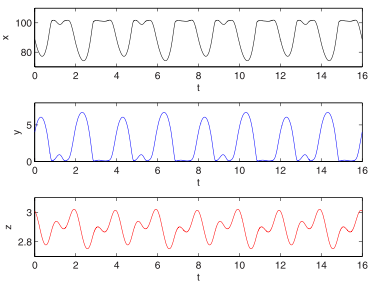

(e)

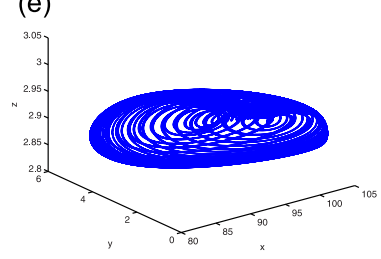

(f)
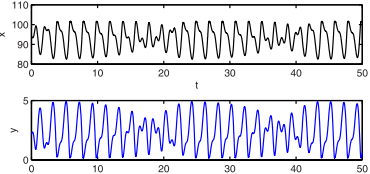

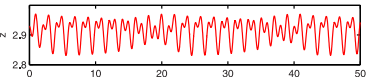

$(\mathrm{g})^{20}$

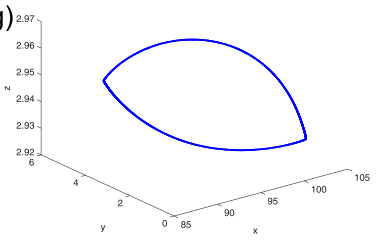

FIG. 7. Phase portrait of different solutions. (a) A stable period-two orbit for $n_{p}=0.026, Y_{p}=0.004, r=0.61$, and $\varepsilon=0.17$. (b) Time series of the stable period-two orbit. (c) A stable period-four orbit for $n_{p}=0.018, Y_{p}=0.001, r=0.594$, and $\varepsilon=0.2$. (d) Time series of the stable period-four orbit. (e) Phase portrait of torus for $n_{p}=0.018, Y_{p}=0.001, r=0.595$, and $\varepsilon=0.08$. (f) Time series of the torus. (g) Poincare section of the torus. 


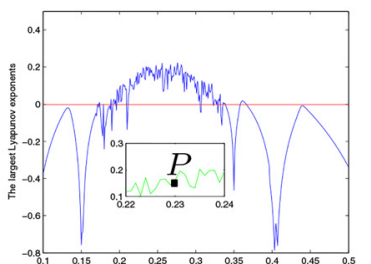

(a)

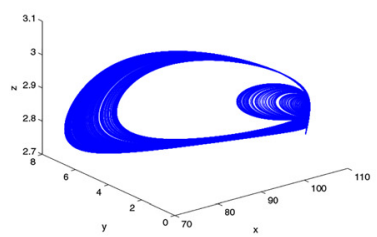

(c)

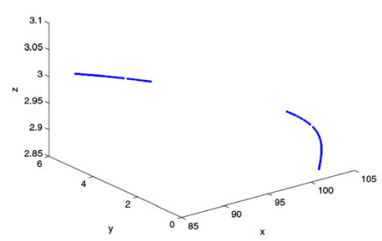

(d)

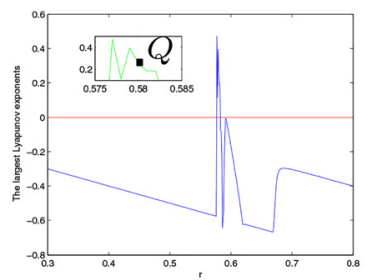

(b)

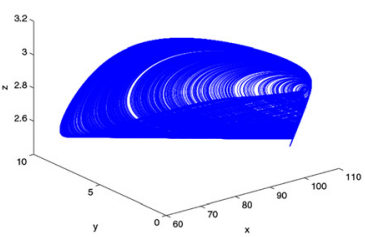

(e)

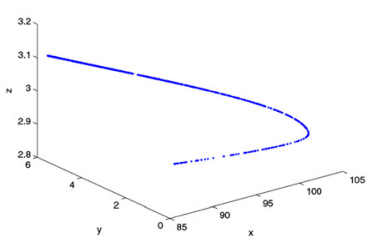

(f)

FIG. 8. (a) Spectrum of largest Lyapunov exponents for $n_{p}=0.018, Y_{p}=0.001$, and $r=0.59$. (b) Spectrum of largest Lyapunov exponents for $n_{p}=0.018$, $Y_{p}=0.001$, and $\varepsilon=0.7$. (c) and (d) Chaotic attractor for $n_{p}=0.018, Y_{p}=0.001, r=0.59, \varepsilon=0.23$, and corresponding Poincaré section. (e) and (f) Chaotic attractor for $n_{p}=0.018, Y_{p}=0.001, r=0.58, \varepsilon=0.7$, and corresponding Poincaré section.

for $n_{p}=0.018, Y_{p}=0.001$, and $r=0.59$, and Fig. $8(\mathrm{~b})$ is the spectrum in the $(\lambda-\mathrm{r})$ plane for $n_{p}=0.018, Y_{p}=0.001$, and $\varepsilon=0.7$. Both of them indicate that chaotic areas exist in some parameter spaces of system (15). On the other hand, some ribbon-like structures with self-similarity in the diagram of Poincaré section also indicate that the corresponding attractor shows chaotic behavior. There are many chaotic attractors in region 4 of Figs. 5 and 6, which are generated by a cascade of period doublings. Figure $8(\mathrm{c})$ is phase portrait of the chaotic attractor for $n_{p}=0.018, Y_{p}=0.001$, $r=0.59$, and $\varepsilon=0.23$; we can find that its Poincaré section has a ribbon-like structure. Besides, the largest Lyapunov exponent of the chaotic attractor in Fig. 8(c) corresponds to the point $P$ in Fig. 8(a), from which one can easily find that it is positive. Figure 8(e) is another example of the chaotic attractor for $n_{p}=0.018, Y_{p}=0.001, r=0.58$, and $\varepsilon=0.7$; the corresponding Poincaré section and largest Lyapunov exponent [the point $Q$ in Fig. 8(b)] indicate that it is chaos.

The results also show that periodical forcing will lead to bistability, where two stable solutions can be converged towards only by taking different initial histories. We can detect the bistability from the bifurcation diagrams, just take the bifurcation diagram Fig. 5 as an example. In fact, a case of bistability is obtained between a stable quasiperiodic and a stable period-two solution for the same group of parameter values, which is generated by the coexistence of NeimarkSacker bifurcation and flip bifurcation in some parameter regions. A pair of period-two orbits appear in region 2, one of them is stable, due to the period-doubling bifurcation $\left(f^{(1)}\right)$ of period-one orbit. On the other hand, a stable quasiperiodic solution also exists in the subregion of region 2 as a result of torus bifurcation. That is to say, we can find a bistability in the subregion of region 2. In Fig. 9, the parameter values of two kinds of solutions are selected as $n_{p}=0.018$, $Y_{p}=0.001, r=0.6$, and $\varepsilon=0.1$. In Fig. 9(a), the initial history of period-two orbit is $x=101.72, y=3.65$, and $z=2.97$. In Fig. 9(c), the initial history of quasiperiodic solution is $x=62.33, y=4.88$, and $z=3$. This clearly shows that there are regions in the $(\mathrm{r}-\varepsilon)$ plane where bistability exists.

\section{DISCUSSION}

A three dimensional microbial continuous culture model with the restrained microbial growth rate function is studied for both the unforced and periodically forced dilution rates. It is shown in this paper that the unforced system undergoes saddle-node bifurcation, generic Hopf bifurcation, and degenerate Hopf bifurcation. For the periodically forced system, we find that the Poincare map of the forced system undergoes Neimark-Sacker bifurcation, flip bifurcation, and fold bifurcation, which lead to the appearance of periodic solutions with different periods and stabilities.

Throughout the results on the unforced and periodically forced systems, we have the following conclusions. (1) Bifurcations of the unforced system can be extended to the periodically forced case as bifurcations of periodic solutions. (2) As the unforced system undergoes Hopf bifurcation, the resulting bifurcations of the periodically forced system are distinct only if the directions of Hopf bifurcation are different (supercritical and subcritical), see Figs. 5 and 6. (3) The periodically forced system can result in more complex dynamics, such as chaos [Figs. 8(c) and 8(e)] and bistability (Fig. 9).

Apart from the theoretical meaning, our results can also explain some biological phenomena in laboratory experiments of microbial continuous culture. ${ }^{4,8,23}$

In Ref. 4, Menzel et al. noticed some oscillations in the process of glycerol continuous fermentation by Klebsiella pneumoniae. They found that the biomass concentration and the specific growth rate $(\mu)$ of cells change periodically with time, and the frequencies of them are nearly the same, see Fig. 1(b) in Ref. 4. Corresponding to the results in Chap. III, when system (1) undergoes a supercritical Hopf bifurcation, a stable periodic solution will appear. That is to say, the biomass, glycerol, and 1,3-PD concentration (variable $x, y$, and $z$, respectively) will vary periodically with a period $T_{0}$. Recalling that the growth rate $(\mu)$ is a function of the variables $y$ and $z$, see expression (2), $\mu$ will change periodically and have the same frequency $1 / T_{0}$ with the oscillation of the biomass concentration. 


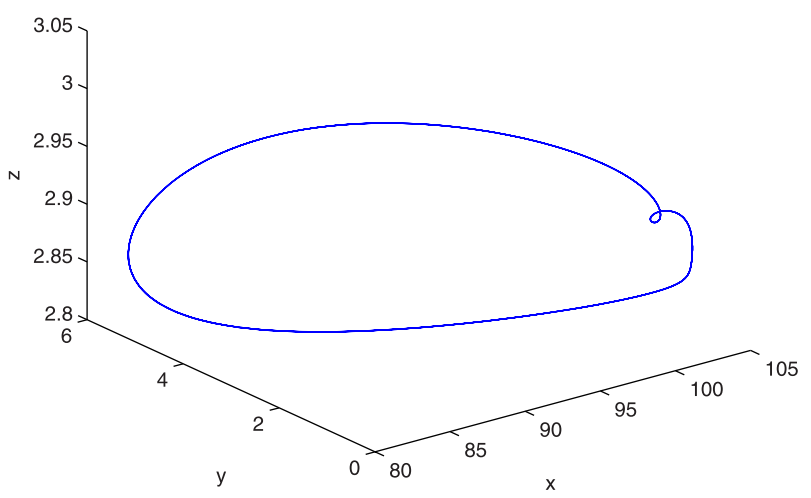

(a)

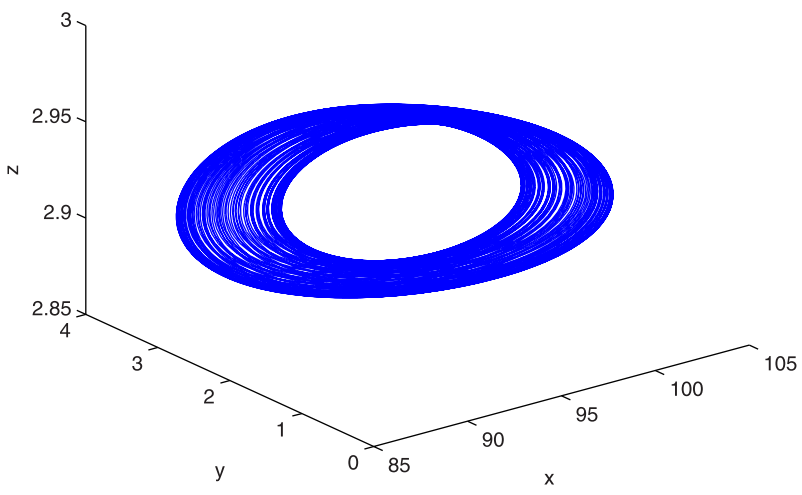

(c)
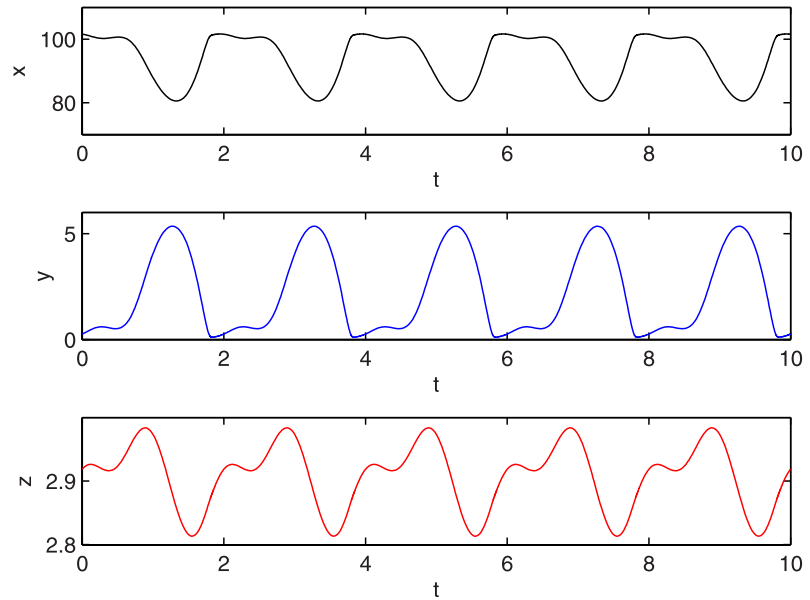

(b)
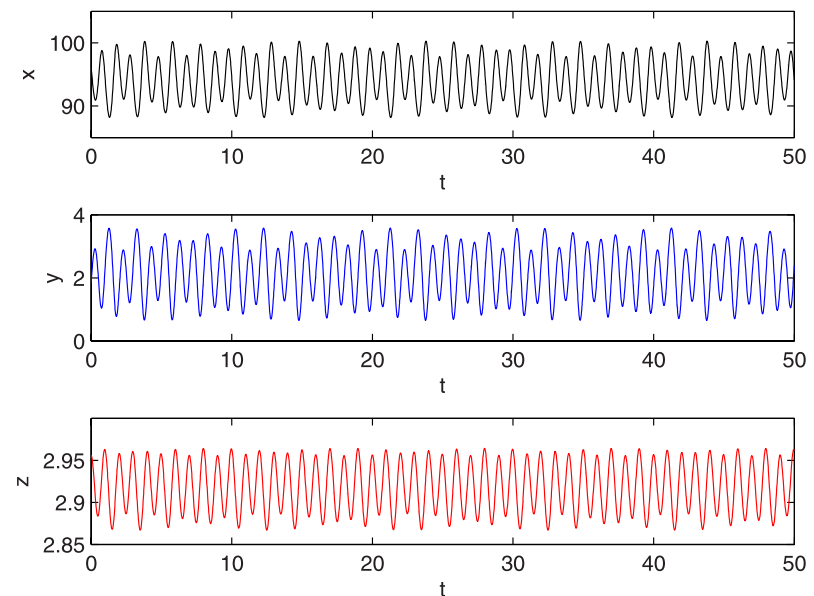

(d)

FIG. 9. Bistability for $n_{p}=0.018, Y_{p}=0.001, r=0.6$, and $\varepsilon=0.1$. (a) A stable period-two orbit. (b) Time series of the stable period-two orbit. (c) A stable quasiperiodic solution. (d) Time series of quasiperiodic solution.

Moreover, the occurrence and disappearance of oscillation of the $\mathrm{CO}_{2}$ concentration are observed in glycerol continuous fermentation, see Fig. 4 in Ref. 4. (The periodic change of the $\mathrm{CO}_{2}$ concentration in the exit gas is often seen as an indicator of oscillations in a cultural process. This is because the oscillations of biomass concentration and $\mathrm{CO}_{2}$ have similar patterns and synchronism.) When system (1) undergoes a subcritical Hopf bifurcation, an unstable periodic orbit and a stable equilibrium will appear, and the generating unstable periodic orbit will tend to the stable equilibrium as $t \rightarrow+\infty$, see Fig. 4(d). That is why the amplitude of the observed oscillation declines with time.

In Ref. 8, Wang et al. detected special oscillations of biomass concentration in the fed-batch fermentation of glycerol to 1,3-PD by Klebsiella pneumoniae. The oscillations can be divided into four distinct phases, cells rapidly grow and the concentration reaches a maximum (phase I), cells cease to grow and the concentration reaches a minimum (phase II), cells grow again and the concentration reaches a second maximum (phase III), and the biomass concentration declines to a second minimum (phase IV), see Fig. 1 in Ref. 8.
During the fed-batch fermentative process, the dilution rate $(D)$ is no longer a constant but a periodic function. For the observed oscillations, we can draw an analogy with the bifurcation results of the periodically forced system (15). A stable period-two solution, which is generated by the Neimark-Sacker bifurcation in the periodically forced system, results in the mentioned phenomena above. To be more clear, we show the time series of a period-two solution in one period, see Fig. 10. Obviously, the period-two solution in Fig. 10 can be divided into four phases, which has two maximum and two minimum in one period. It gives a clue for the appearance of oscillations in Ref. 8.

In Ref. 23, Grosz and Stephanopoulos observed the multiply steady state in a microbial continuous culture process, where the steady-state solution can change from the upper to the lower solution branches. The different stable equilibria in this paper correspond to the observed multiply steady state, see Lemma 1 and Theorem 2. Moreover, the bistability generated by bifurcations in the forced system can also illustrate the multiply steady state, where two stead states can be reached just by taking different initial 


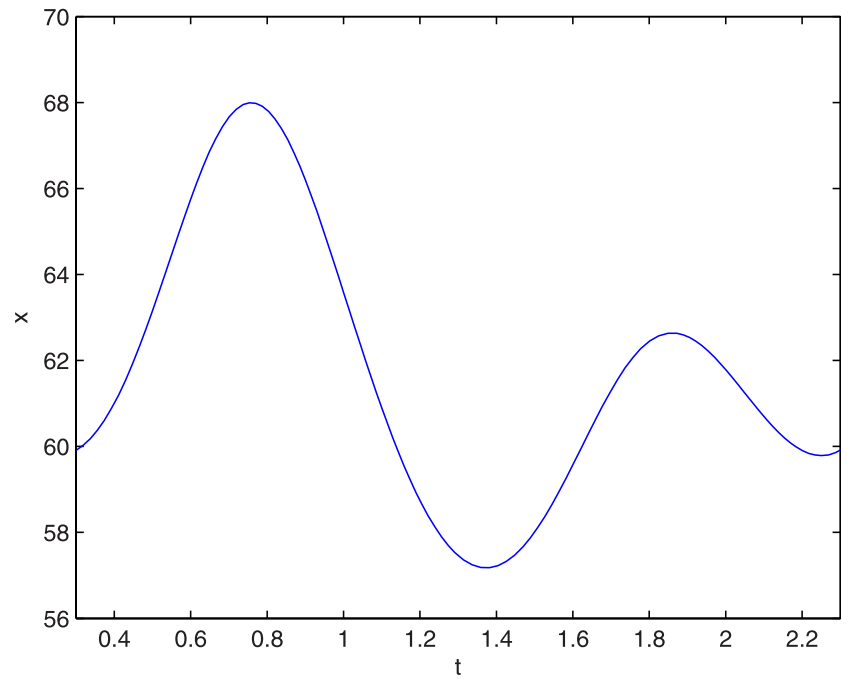

FIG. 10. Time series of a period-two solution in one period, where the parameter values are fixed as those in Fig. 7(a).

histories. When the system undergoes Hopf bifurcation or flip bifurcation, the corresponding stable solution becomes unstable. Then, the generating unstable solution will tend to another stable solution as time changes, that is why the steady-state solution changes to other branches.

Besides, some unusual oscillations, which have irregular period or no period completely, are discovered in glycerol fermentation, see Figs. 3(c) and 3(d) in Ref. 4 and Fig. 2 in Ref. 8. We suggest that these oscillations could be caused by the quasiperiodic solutions or chaos in a short time. In fact, the chaotic attractors obtained by a cascade of period doublings in our paper are not absolutely ruleless. If the time series of the chaos are considered in a short time, we find that it is an oscillation with an irregular period, see Fig. 11. In a word, the limit cycles, periodic solutions, quasiperiodic solutions, and chaotic attractor obtained by different bifurcations are mechanisms of the oscillations in a microbial continuous culture process.

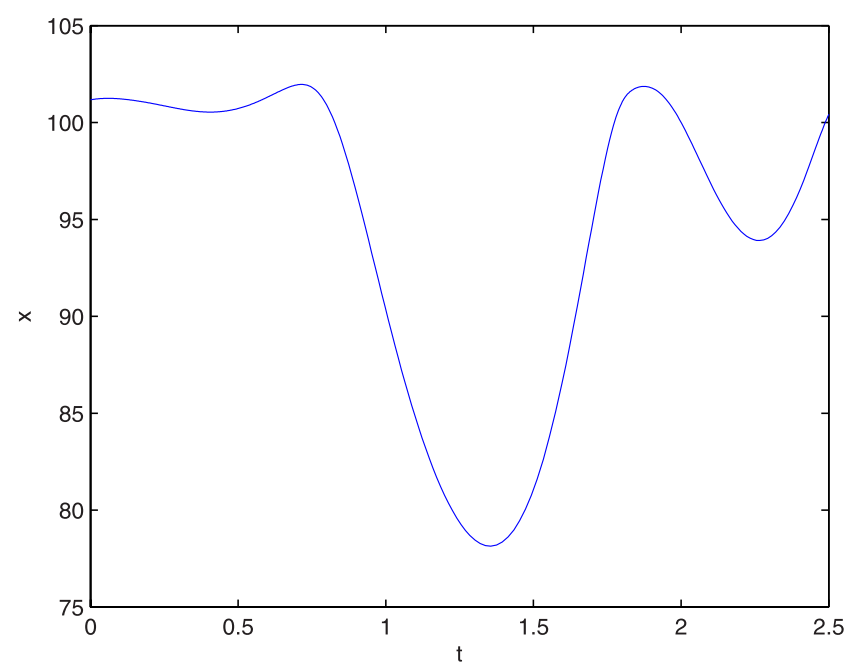

FIG. 11. Time series of a chaotic attractor in a short time, where the parameter values are fixed as those in Fig. 8(c).

\section{ACKNOWLEDGMENTS}

This work was supported by the NSFC (11771407) project, the Innovation Research Team of Science and Technology of Henan Province (17IRTSTHN007), the Plan for Scientific Innovation Talent of Henan Province (164200510011), the Opening fund of State Key Laboratory of Nonlinear Mechanics (LNM201710), and the National Key Research and Development Program of China (2017YFB0702500).

${ }^{1}$ U. Witt, R. J. Müller, J. Augusta, H. Widdecke, and W. D. Deckwer, "Synthesis,properties and biodegradability of polyesters based on 1,3-propanediol," Macromol. Chem. Phys. 195, 793-802 (1994).

${ }^{2}$ H. Biebl, "Glycerol fermentation of 1, 3-propanediol by clostridium butyricum. measurement of product inhibition by use of a ph-auxostat," Appl. Microbiol. Biotechnol. 35, 701-705 (1991).

${ }^{3}$ Y. Q. Sun, W. T. Qi, H. Teng, Z. L. Xiu, and A. Zeng, "Mathematical modeling of glycerol fermentation by klebsiella pneumoniae: Concerning enzyme-catalytic reductive pathway and transport of glycerol and 1,3-propanediol across cell membrane," Biochem. Eng. J. 38, 22-32 (2008).

${ }^{4}$ K. Menzel, A. P. Zeng, H. Biebl, and W. D. Deckwe, "Kinetic, dynamic, and pathway studies of glycerol metabolism by klebsiella pneumoniae in anaerobic continuous culture: I. The phenomena and characterization of oscillation and hysteresis," Biotechnol. Bioeng. 52, 549-560 (1996).

${ }^{5}$ A. P. Zeng, A. Ross, H. Biebl, C. Tag, B. Gönzel, and W. Deckwer, "Multiple product inhibition and growth modeling of clostridium butyricum and klebsiella pneumoniae in glycerol fermentation," Biotechnol. Bioeng. 44, 902-911 (1994).

${ }^{6}$ C. X. Gao, E. M. Feng, Z. T. Wang, and Z. L. Xiu, "Parameters identification problem of the nonlinear dynamical system in microbial continuous cultures," Appl. Math. Comput. 169, 476-484 (2005).

${ }^{7}$ J. X. Ye, E. M. Feng, H. S. Lian, and Z. Xiu, "Existence of equilibrium points and stability of the nonlinear dynamical system in microbial continuous cultures," Appl. Math. Comput. 207, 307-318 (2009).

${ }^{8}$ W. Wang, J. B. Sun, M. Hartlep, W. D. Deckwer, and A. P. Zeng, "Combined use of proteomic analysis and enzyme activity assays for metabolic pathway analysis of glycerol fermentation by klebsiella pneumoniae," Biotechnol. Bioeng. 83, 525-536 (2003).

${ }^{9}$ D. E. Contois, "Kinetics of bacterial growth: Relationship between population density and specific growth rate of continuous cultures," J. Gen. Microbiol. 21, 40-50 (1959).

${ }^{10} \mathrm{~J}$. F. Andrews, "A mathematical model for the continuous culture of microorganisms utilizing inhibitory substrates," Biotechnol. Bioeng. 10, 703-723 (1968)

${ }^{11}$ S. Papanikolaou, M. Fick, and G. Aggelis, "The effect of raw glycerol concentration on the production of 1,3-propanediol by clostridium butyricum," J. Chem. Technol. Biotechnol. 79, 1189-1196 (2004).

${ }^{12}$ A. P. Zeng and W. D. Deckwer, "A kinetic model for substrate and energy consumption of microbial growth under substrate-sufficient conditions," Biotechnol. Prog. 11, 71-79 (1995).

${ }^{13}$ J. Chen, J. C. Huang, S. G. Ruan, and J. H. Wang, "Bifurcations of invariant tori in predator-prey models with seasonal prey harvesting," SIAM J. Appl. Math. 73, 1876-1905 (2013).

${ }^{14}$ J. M. Gonzlez-Miranda, "On the effect of circadian oscillations on biochemical cell signaling by nf-b," J. Theor. Biol. 335, 283-294 (2013).

${ }^{15}$ J. L. Ren and X. P. Li, "Bifurcations in a seasonally forced predator-prey model with generalized holling type iv functional response," Int. J. Bifurcation Chaos 26, 1650203 (2016).

${ }^{16}$ Y. A. Kuznetsov, Elements of Applied Bifurcation Theory, 2nd ed., edited by J. E. Marsden and L. Sirovich (Springer-Verlag, New York, 1998).

${ }^{17}$ F. Dumortier, R. Roussarie, J. Sotomayor, and H. Zoladek, Bifurcations of Planar Vector Fields, Nilpotent Singularities and Abelian Integrals, Lecture Notes in Mathematics (Springer-Verlag, New York, 1991).

${ }^{18}$ C. H. Shan, Y. F. Yi, and H. P. Zhu, "Nilpotent singularities and dynamics in an sir type of compartmental model with hospital resources," J. Differ. Equations 260, 4339-4365 (2016). 
${ }^{19}$ E. J. Doedel and B. E. Oldeman, See http://cmvl.cs.concordia.ca/auto for Auto-07p: continuation and bifurcation software for ordinary differential equations.

${ }^{20}$ J. L. Ren and L. P. Yu, "Codimension-two bifurcation, chaos and control in a discrete-time information diffusion model," J. Nonlinear Sci. 26, 1895-1931 (2016).

${ }^{21}$ B. Li and Z. M. He, "1:2 and 1:4 resonances in a two-dimensional discrete Hindmarsh-Rose model,” Nonlinear Dyn. 79, 705-720 (2015).
${ }^{22}$ M. T. Rosenstein, J. J. Collins, and C. J. D. Luca, "A practical method for calculating largest Lyapunov exponents from small data sets," Phys. D: Nonlinear Phenom. 65, 117-134 (1993).

${ }^{23}$ R. Grosz and G. Stephanopoulos, "Physiological, biochemical, and mathematical studies of micro-aerobic continuous ethanol fermentation by saccharom yces cerevisiae. I. Hysteresis, oscillations, and maximum specific ethanol productivities in chemostat culture," Biotechnol. Bioeng. 36, 1006-1019 (1990). 